\title{
Beryllium-10 production in gaseous protoplanetary disks and implications for the astrophysical setting of refractory inclusions
}

\author{
Emmanuel Jacquet
}

\begin{abstract}
Muséum national d'Histoire naturelle, IMPMC, Département Origines et Evolution, 57 rue Cuvier, 75005 Paris, France e-mail: emmanuel.jacquet@mnhn.fr
\end{abstract}

Received 30 November 2018 / Accepted 7 March 2019

\begin{abstract}
Calcium-aluminum-rich inclusions (CAIs), the oldest known solids of the solar system, show evidence for the past presence of shortlived radionuclide beryllium-10, which was likely produced by spallation during protosolar flares. While such ${ }^{10}$ Be production has hitherto been modeled at the inner edge of the protoplanetary disk, I calculate here that spallation at the disk surface may reproduce the measured ${ }^{10} \mathrm{Be} /{ }^{9} \mathrm{Be}$ ratios at larger heliocentric distances. Beryllium-10 production in the gas prior to CAI formation would dominate that in the solid. Interestingly, provided the Sun's proton to X-ray output ratio does not decrease strongly, ${ }^{10} \mathrm{Be} /{ }^{9} \mathrm{Be}$ at the CAI condensation front would increase with time, explaining the reduced values in a (presumably early) generation of CAIs with nucleosynthetic anomalies. CAIs thus need not have formed very close to the Sun and may have condensed at 0.1-1 AU where sufficiently high temperatures originally prevailed.
\end{abstract}

Key words. Sun: flares - meteorites, meteors, meteoroids - accretion, accretion disks - stars: protostars - cosmic rays $-\mathrm{X}$-rays: stars

\section{Introduction}

Primitive meteorites, or chondrites, are conglomerates of solids that formed and accreted in the solar protoplanetary disk. Among those, the oldest are the refractory inclusions, comprising calcium-aluminium-rich inclusions (CAIs) and amoeboid olivine aggregates (AOA) (e.g., MacPherson 2014; Krot et al. 2004). Thermodynamic calculations predict that CAIs should be the first condensates in a cooling gas of solar composition (Grossman 2010; Davis \& Richter 2014). Since the required temperatures would be in the range 1500-2000 K (Wooden et al. 2007), it is widely assumed that refractory inclusions formed close to the Sun, but precisely how close and in what astrophysical setting remains unclear (Wood 2004; Jacquet 2014).

An important clue in this respect may be provided by beryllium-10, a short-lived radionuclide decaying into boron-10 with a half-life of $1.5 \mathrm{Ma}$, and which, unlike other known extinct radionuclides such as aluminum-26, is not produced by stellar nucleosynthesis but may be formed through spallation by energetic particles (Davis \& McKeegan 2014; Lugaro et al. 2018). Indeed, since the original discovery by McKeegan et al. (2000), all CAIs with analytically suitable Be/B have shown ${ }^{10} \mathrm{~B} /{ }^{11} \mathrm{~B}$ excesses correlated therewith. The slopes of the resulting isochrons translate into initial (i.e., upon the last isotopic equilibration) ${ }^{10} \mathrm{Be} /{ }^{9} \mathrm{Be}$ ratios averaging $\sim 6 \times 10^{-4}$ in $\mathrm{CV}$ chondrite (type A and B) CAIs (Davis \& McKeegan 2014), with less than a factor of two spread. Yet systematically lower values around $3 \times 10^{-4}$ and $5 \times 10^{-4}$ have been found for two $\mathrm{CV}$ chondrite FUN CAIs (fractionated and unknown nuclear effects; MacPherson et al. 2003; Wielandt et al. 2012) and platy hibonite crystals (PLAC) in CM chondrites (Liu et al. 2009, 2010), respectively, and, conversely, values up to $10^{-2}$ have been found for Isheyevo CAI 411 (Gounelle et al. 2013) and more recently, fine-grained group II CV chondrite CAIs (Sossi et al.
2017). The overall spread, along with correlated ${ }^{50} \mathrm{~V}$ excesses (also ascribed to spallation) in the latter objects (Sossi et al. 2017 ), is difficult to reconcile with simple ${ }^{10} \mathrm{Be}$ inheritance from the (galactic cosmic ray-irradiated) protosolar cloud (Desch et al. 2004), which should be largely homogeneous, and argues in favor of local production in the disk following flares from the young Sun (Gounelle et al. 2001, 2006, 2013; Sossi et al. 2017), such as those manifested in X-rays by present-day protostars (e.g., Feigelson et al. 2002; Wolk et al. 2005; Preibisch et al. 2005; Telleschi et al. 2007; Güdel et al. 2007; Bustamante et al. 2016) or evidence for enhanced ionization in some protostellar envelopes (Ceccarelli et al. 2014; Favre et al. 2017, 2018).

Since the energetic protons would not penetrate further than $\sim 690 \mathrm{~kg} \mathrm{~m}^{-2}$ (Umebayashi \& Nakano 1981) into the gas, Sossi et al. (2017) suggested that CAIs spent a few thousand orbits at the inner edge of the protoplanetary disk, similar to earlier studies that had adopted the framework of the X-wind scenario (Lee et al. 1998; Shu et al. 2001; Gounelle et al. 2001). However, among the objections to the latter discussed by Desch et al. (2010), a general issue is that CAI-forming temperatures would be expected significantly further outward (0.1-1 AU), because of local dissipation of turbulence. If nonetheless CAIs all went somehow through this narrow region and escaped accretion to the Sun or ejection from the solar system, they would be essentially "lucky" foreign material in the chondrites that incorporated them. Yet CAIs are present in carbonaceous chondrites at about the abundances predicted by in situ condensation (Jacquet et al. 2012). Also, although (non-CI) carbonaceous chondrites are enriched in refractory elements relative to CI chondrites, this cannot be ascribed to simple CAI addition to CAI-free CI chondritic material for should the CAIs be mentally subtracted, these chondrites would have subsolar refractory element abundances (Hezel et al. 2008), not even considering those CAIs that were converted into chondrules (Misawa \& Nakamura 1988; 


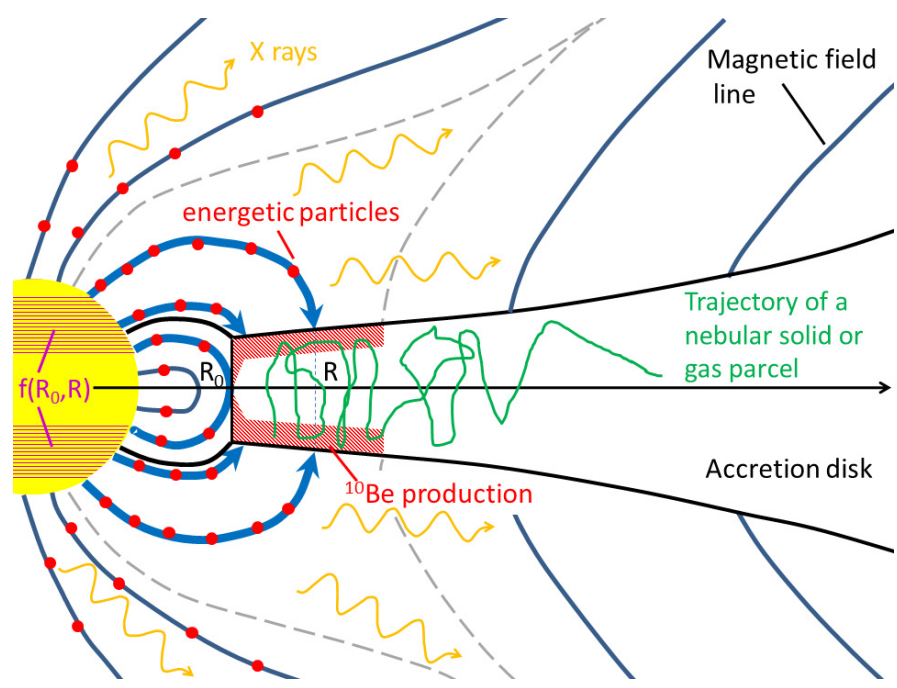

Fig. 1. Sketch of the scenario of ${ }^{10} \mathrm{Be}$ production developed in this paper. Solar cosmic rays cause spallation reactions in the upper layers of the disk, in the gas and/or condensates, within a region (outlined by a dashed separatrix) where magnetic field lines originating from the proto-Sun and its outskirts hit the disk. The field and particle emission geometries are purely illustrative and do not detract from the generality of the formalism.

Jones \& Schilk 2009; Metzler \& Pack 2016; Ebert \& Bischoff 2016; Jacquet \& Marrocchi 2017; Marrocchi et al. 2018). Isotopic systematics for elements of different volatilities also indicate a need for a non-refractory isotopically CAI-like component in chondrites (e.g. Nanne et al. 2019). So there is evidence for a genetic relationship between CAIs and (part of) their host carbonaceous chondrites, which argues against an origin at the disk inner edge, whose contribution to distant chondritic matter would likely be minor.

However, the inner edge of the disk is not the only region where CAIs or their precursors could have been exposed to energetic solar protons. CAIs floating further out in the disk would at times have reached the upper layers of the disk. While such excursions may individually incur modest proton fluences, their cumulative contributions might explain the total ${ }^{10} \mathrm{Be}$ evidenced in CAIs. Moreover, prior to CAI condensation, the gas exposed at the surface of the disk may also have undergone spallation and passed on the then-produced ${ }^{10} \mathrm{Be}$ to the later formed condensates. The purpose of this paper is to analytically calculate the amount of beryllium-10 produced by such channels and thence evaluate the viability of having CAIs form at 0.1-1 AU from the young Sun against their irradiation record. Figure 1 provides a sketch of the overall scenario and setting. After several generalities in Sect. 2, I will express the ${ }^{10} \mathrm{Be} /{ }^{9} \mathrm{Be}$ ratio in the disk and free-floating solid CAIs in Sect. 3. I then discuss the numerical evaluations thereof and their implications in Sect. 4 before concluding in Sect. 5 .

\section{Prolegomena}

\subsection{Disk model}

I consider a protoplanetary disk in a cylindrical coordinate system with heliocentric distance $R$. The inner regions can be described under the steady-state approximation so long as the evolution timescales of the system are long compared to their local viscous timescale

$$
\begin{aligned}
t_{\mathrm{vis}}(R) & \equiv \frac{R^{2}}{v} \\
& =0.02 \mathrm{Ma}\left(\frac{R}{0.5 \mathrm{UA}}\right)^{1 / 2}\left(\frac{1500 \mathrm{~K}}{T}\right)\left(\frac{10^{-3}}{\alpha}\right),
\end{aligned}
$$

where $v=\alpha c_{\mathrm{s}}^{2} / \Omega_{\mathrm{K}}$ is the effective turbulent viscosity, $\alpha$ the dimensionless turbulence parameter (e.g., Jacquet 2013), $c_{\mathrm{s}}=$ $\sqrt{k_{\mathrm{B}} T / m}$ the isothermal sound speed with $k_{\mathrm{B}}$ the Boltzmann constant, $T$ the temperature and $m=3.9 \times 10^{-27} \mathrm{~kg}$ the mean molecular mass, and $\Omega_{\mathrm{K}}$ the Keplerian angular velocity. Since $t_{\text {vis }}(R)$ is also much shorter than the half-life of ${ }^{10} \mathrm{Be}$, I will neglect its decay during the CAI formation epoch. This is consistent with the short timescales (no more than a few hundreds of millenia) of CAI formation and (isotopically resetting) reheating events (e.g., MacPherson 2014), which should have ended after the last production of ${ }^{10} \mathrm{Be}$ so as to account for the isochron behavior of the Be-B system in each CAI (that is, with ${ }^{10} \mathrm{~B}$ excesses scaling with $\mathrm{Be}$ rather than the target nuclides leading to its parent; see next subsection).

If infall from the parental cloud can be neglected in the inner region, the disk mass accretion rate $\dot{M}=-2 \pi R \Sigma u_{\mathrm{R}}$ (with $\Sigma$ the disk surface density and $u_{\mathrm{R}}$ the net, turbulence-averaged, gas radial velocity) is uniform and obeys

$\dot{M}\left(1-\sqrt{\frac{R_{0}}{R}}\right)=3 \pi \Sigma v$,

or, equivalently,

$u_{\mathrm{R}}=-\frac{3 v}{2 R\left(1-\sqrt{R_{0} / R}\right)}$,

where we have assumed that the stress vanishes at the disk inner edge $R_{0}$ (e.g., Balbus \& Hawley 1998). When calculating ${ }^{10} \mathrm{Be} /{ }^{9} \mathrm{Be}$ in later sections, I shall show expressions for a general $\dot{M}(R)$ before specializing to the case of uniform mass accretion rate. Appendix A explores the corrections of infall to Eq. (2).

If I inject the temperature due to viscous dissipation of turbulence (e.g., Appendix A of Jacquet et al. 2012) in Eq. (2), I obtain

$$
\begin{aligned}
T= & \left(\frac{3 \kappa m \dot{M}^{2}\left(1-\sqrt{R_{0} / R}\right)^{2} \Omega_{\mathrm{K}}^{3}}{128 \pi^{2} \sigma_{\mathrm{SB}} k_{\mathrm{B}} \alpha}\right)^{1 / 5} \\
= & 1200 \mathrm{~K}\left(\frac{\kappa}{10^{-2} \mathrm{~m}^{2} \mathrm{~kg}^{-1}}\right)^{1 / 5}\left(\frac{10^{-3}}{\alpha}\right)^{1 / 5}\left(1-\sqrt{\frac{R_{0}}{R}}\right)^{2 / 5} \\
& \times\left(\frac{0.5 \mathrm{AU}}{R}\right)^{9 / 10}\left(\frac{\dot{M}}{10^{-7} M_{\odot} / a}\right)^{2 / 5},
\end{aligned}
$$

with $\kappa$ the specific Rosseland mean opacity and $\sigma_{\mathrm{SB}}$ the StefanBoltzmann constant. This confirms that CAI-forming temperatures may be reached at a fraction of an AU for $\dot{M} \gtrsim 10^{-7} M_{\odot} / a$.

\section{2. ${ }^{10}$ Be production rate}

The local production rate of ${ }^{10} \mathrm{Be}$ by spallation normalized to ${ }^{9}$ Be may be written as (e.g., Sossi et al. 2017)

$$
\left(\frac{{ }^{10} \mathrm{Be}}{{ }^{9} \mathrm{Be}}\right)_{\operatorname{tg}}=\int_{0}^{+\infty} \mathrm{d} E \sigma_{k, i}(E)\left(\frac{k}{\mathrm{Be}}\right)_{\operatorname{tg}} \int \mathrm{d} \Omega I(i, E, \Omega),
$$


with $\sigma_{k, i}$ the ${ }^{10} \mathrm{Be}$ production cross section for target (tg) nuclide $k$ and cosmic ray species $i$ (with energy per nucleon $E$ ) and $I(i, E, \Omega)$ the corresponding (orientation-dependent) monoenergetic cosmic ray specific intensity (defined by number and not energy, as in Gounelle et al. 2001). Here and throughout, all isotopic or elemental ratios are atomic and the Einstein convention for summation over the repeated indices $k$ and $i$ is adopted. I assume that the incoming cosmic rays have a solar composition (e.g. ${ }^{4} \mathrm{He} / \mathrm{H}=0.1$; but see Mewaldt et al. 2007 for details on contemporaneous solar energetic particles), and obey a power law $\propto$ $E^{-p}$ for $E \geq E_{10} \equiv 10 \mathrm{MeV}$ upon arrival on the disk. I will ignore the contribution of secondary neutrons, despite their comparable cross sections (e.g., Leya \& Masarik 2009) and larger attenuation columns. This is because a dilute medium such as the surface of the disk will let free decay thwart significant accumulation of neutron flux (Umebayashi \& Nakano 1981). However, based on this latter work, this is only marginally true (see Eq. (C.5)), so the production rates given here should be strictly viewed as a lower bounds.

In the following sections, we will be interested in the density $(\rho)$-weighted vertical average of the production rate at a given heliocentric distance,

$\left\langle\left(\frac{{ }^{10} \mathrm{Be}}{{ }^{9} \mathrm{Be}}\right)\right\rangle_{\operatorname{tg} \rho} \equiv \frac{1}{\Sigma} \int_{0}^{\Sigma} \mathrm{d} \Sigma^{\prime}\left(\frac{{ }^{10} \mathrm{Be}}{{ }^{9} \mathrm{Be}}\right)_{\operatorname{tg}} \approx \frac{2}{\Sigma} \int_{0}^{+\infty} \mathrm{d} \Sigma^{\prime}\left(\frac{{ }^{10} \mathrm{Be}}{{ }^{9} \mathrm{Be}}\right)_{\operatorname{tg},+}$,

where $\Sigma^{\prime}$ is the column density integrated vertically from the upper surface and the +subscript in the final equality denotes restriction to cosmic rays from the upper $\operatorname{side}^{1}$, assuming $\Sigma$ is much larger than the attenuation column $\Sigma_{p, i}(E)$ of the cosmic rays, defined here as

$\Sigma_{p, i}(E) \equiv \frac{1}{I_{+}(i, E, \Omega ; 0)} \int_{0}^{+\infty} \mathrm{d} \Sigma^{\prime \prime} I_{+}\left(i, E, \Omega ; \Sigma^{\prime \prime}\right)$,

where $\Sigma^{\prime \prime}$ is the actually traversed column density. If I envision a cosmic ray beam (with the particles gyrating along a magnetic field line) penetrating the disk with a grazing angle $\phi$ and ignore secondary particles, $\Sigma^{\prime \prime}=\Sigma^{\prime} /(\mu \sin \phi)$ with $\mu$ the cosine of the pitch angle of the cosmic ray with respect to the magnetic field line (Padovani et al. 2018). I thus obtain

$\left\langle\left(\frac{{ }^{10} \mathrm{Be}}{{ }^{9} \mathrm{Be}}\right)_{\operatorname{tg}}\right\rangle_{\rho}=\frac{2}{\Sigma} \int_{0}^{+\infty} \mathrm{d} E \sigma_{k, i}(E) \Sigma_{p, i}(E)\left(\frac{k}{\mathrm{Be}}\right)_{\mathrm{tg}}\left(\frac{i}{\mathrm{H}}\right)_{\mathrm{CR}} \frac{\mathrm{d} \boldsymbol{F}_{H}}{\mathrm{~d} E} \cdot \boldsymbol{n}$,

where $\boldsymbol{n}$ is the downward-directed unit vector normal to the disk surface and $\mathrm{d} \boldsymbol{F}_{H} / \mathrm{d} E$ the incoming differential proton number vector flux. If I scale the latter to the incoming $>10 \mathrm{MeV}$ nucleon $^{-1}$ energy vector flux $\boldsymbol{F}_{10}$ and extract $\mathrm{O}$ as a proxy for all ${ }^{10} \mathrm{Be}$-producing target nuclides, Eq. (8) can be further manipulated to finally yield

$\left\langle\left(\frac{{ }^{10} \mathrm{Be}}{{ }^{9} \mathrm{Be}}\right)_{\operatorname{tg}}\right\rangle_{\rho}=\frac{K_{\mathrm{p}} L_{10}}{2 \pi R \Sigma}\left(\frac{\mathrm{O}}{\mathrm{Be}}\right)_{\operatorname{tg}} \frac{\partial f\left(R_{0}, R\right)}{\partial R}$

with

$K_{\mathrm{p}} \equiv(p-2) E_{10}^{p-2}\left(\frac{k}{\mathrm{O}}\right)_{\mathrm{tg}}\left(\frac{i}{\mathrm{H}}\right)_{\mathrm{CR}} \int_{0}^{+\infty} \mathrm{d} E \sigma_{k, i}(E) \Sigma_{p, i}(E) E^{-p}$,

1 Assuming symmetry of irradiation about the midplane, although this is not a prerequisite to the final result (Eq. (9)).

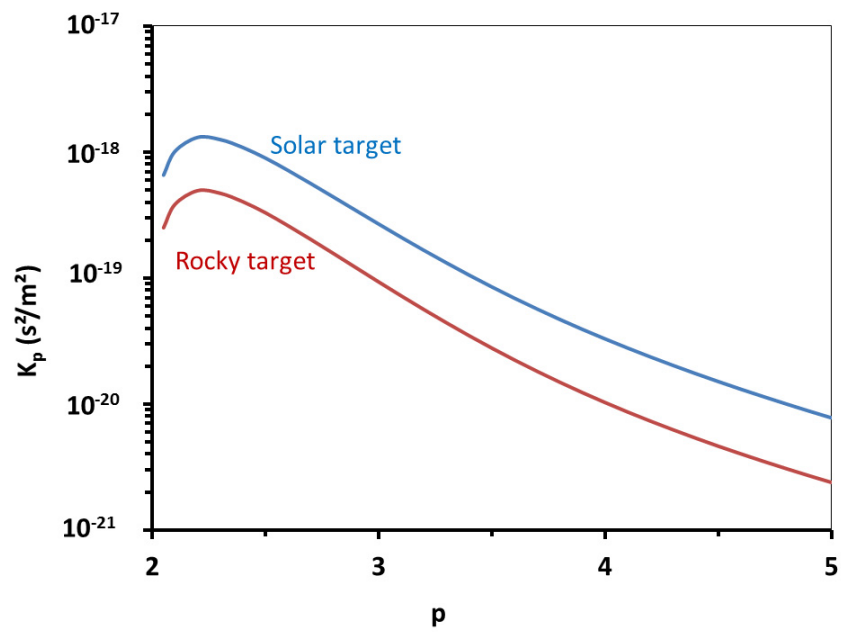

Fig. 2. $K_{\mathrm{p}}$ as a function of the power-law exponent of the incoming cosmic ray energy distribution, for a solar (without possible implantation contributions) and a rocky target (see Sects. 3.1 and 3.2, respectively).

which is plotted in Fig. 2 (see Appendix B for further details on its calculation and data sources). Here, $f(a, b)$ is the fraction of the total $>10 \mathrm{MeV}$ nucleon $^{-1}$ energy luminosity $L_{10}$ that reaches the disk between heliocentric distances $a$ and $b$. The latter depends on the geometry of energetic particle emission and the magnetic field configuration around the young Sun, which are largely unknown (protosolar corona, star-disk fields, or disk fields; Feigelson et al. 2002; Donati \& Landstreet 2009; Padovani et al. 2016). For reference, in the case of a geometrically flared disk, an isotropic point source with straight trajectories would yield $f\left(R_{0}, R\right)=H_{\text {spall }} / R$ with $H_{\text {spall }}$ the height of the spallation layer above the midplane (see Appendix $\mathrm{C}$ for an estimate). For illustration in the plots of the next section, I will use a form of $f$ assuming that the outgoing energy flux density of the energetic particles is uniform over the Sun-centered sphere of radius $R_{0}$ and that the magnetic field is approximately dipolar (with a magnetic moment along the rotation axis of the disk) for field lines interior to a maximum irradiation distance $R_{\max }$. This yields ${ }^{2}$

$f\left(R_{0}, R\right)=\max \left(\sqrt{1-\frac{R_{0}}{R}}, \sqrt{1-\frac{R_{0}}{R_{\max }}}\right)$.

However, in the main text, I will keep general expressions as functions of $f$. This parameterization of our ignorance will turn out to be quite useful in the calculation.

A means of comparison with previous models invoking irradiation of bare solids (e.g., Lee et al. 1998; Gounelle et al. 2006; Sossi et al. 2017) is to divide the rate given by Eq. (9) by that in a target of the same composition exposed to the same unattenuated proton flux. Assuming isotropic distribution of cosmic rays (in each half-space along the field line), this gives

$\frac{{ }^{10} \mathrm{Be}}{{ }^{10} \dot{\mathrm{Be}}_{\text {bare }}}=\frac{\left\langle\Sigma_{p}(E)\right\rangle_{\sigma E^{-p}} \sin \phi}{\Sigma}$

2 If we follow a field line from $(r, \theta)=\left(R_{0}, \theta_{0}\right)$ in polar coordinates, we have

$\frac{\mathrm{d} r}{r \mathrm{~d} \theta}=\frac{B_{r}}{B_{\theta}}=\frac{2 \cos \theta}{\sin \theta}$,

so that upon crossing the midplane $r=R=R_{0} / \sin ^{2} \theta_{0}$ with $f\left(R_{0}, R\right)=$ $\cos \theta_{0}$. 
with

$$
\begin{aligned}
\left\langle\Sigma_{p}(E)\right\rangle_{\sigma E^{-p}} \equiv & \left(\frac{k}{\mathrm{O}}\right)_{\mathrm{tg}}\left(\frac{i}{\mathrm{H}}\right)_{\mathrm{CR}} \int_{0}^{+\infty} \mathrm{d} E \sigma_{k, i}(E) \Sigma_{p, i}(E) E^{-p} \\
& /\left[\left(\frac{k}{\mathrm{O}}\right)_{\mathrm{tg}}\left(\frac{i}{\mathrm{H}}\right)_{\mathrm{CR}} \int_{0}^{+\infty} \mathrm{d} E \sigma_{k, i}(E) E^{-p}\right],
\end{aligned}
$$

which (for a refractory target) is $90 \mathrm{~kg} \mathrm{~m}^{-2}$ for $p=2.5$ typical of gradual flares favored by Gounelle et al. (2013) and Sossi et al. (2017), and $5 \mathrm{~kg} \mathrm{~m}^{-2}$ for $p=4$ commensurate with impulsive flares (with subdominant fluences in the present-day Sun; Desai \& Giacalone 2016). Obviously, for $\Sigma \gg 10^{4} \mathrm{~kg} \mathrm{~m}^{-2}$ (the Minimum Mass Solar Nebula at 1 AU; Hayashi 1981) this calls for (intermittent) irradiation timescales $2 \pm 1$ orders of magnitude above the centuries calculated by Sossi et al. (2017). As alluded to in the Introduction, the transport timescale $t_{\mathrm{vis}}$ may, however, provide the correct order of magnitude. The following section undertakes to calculate more precisely the net ${ }^{10} \mathrm{Be}$ abundances produced in gas and solids and during radial transport.

\section{3. ${ }^{10} \mathrm{Be}$ abundances}

\subsection{Bulk ${ }^{10} \mathrm{Be} /{ }^{9} \mathrm{Be}$ in the disk}

In this subsection, I calculate the average ${ }^{10} \mathrm{Be} /{ }^{9} \mathrm{Be}$ of the disk as a function of heliocentric distance, irrespective of its (temperature-dependent) physical state (condensation fraction) to which spallation reactions are insensitive. I assume solar abundances throughout the inner disk owing to tight coupling of the condensates with the gas (e.g., Jacquet et al. 2012).

A steady-state gradient of ${ }^{10} \mathrm{Be} /{ }^{9} \mathrm{Be}$ should arise in the disk because production of ${ }^{10} \mathrm{Be}$ is balanced by loss to the Sun by accretion. The transport equation reads

$$
\begin{gathered}
\frac{1}{R} \frac{\partial}{\partial R}\left[R\left(\Sigma u_{\mathrm{R}} c_{\mathrm{Be}}\left(\frac{{ }^{10} \mathrm{Be}}{{ }^{9} \mathrm{Be}}\right)_{\text {disk }}-D_{\mathrm{R}} \Sigma \frac{\partial}{\partial R}\left(c_{\mathrm{Be}}\left(\frac{{ }^{10} \mathrm{Be}}{{ }^{9} \mathrm{Be}}\right)_{\text {disk }}\right)\right)\right] \\
=\Sigma c_{\mathrm{Be}}\left\langle\left(\frac{\bullet{ }^{10} \mathrm{Be}}{{ }^{9} \mathrm{Be}}\right)_{\text {disk }}\right\rangle_{\rho}=\frac{K_{p \text {, disk }} L_{10} c_{\mathrm{O}}}{2 \pi R} \frac{\partial f\left(R_{0}, R\right)}{\partial R},
\end{gathered}
$$

with $c_{\mathrm{Be}, \mathrm{O}}$ the $\mathrm{Be}, \mathrm{O}$ concentration in number per unit mass of solar gas and $D_{\mathrm{R}}$ the turbulent diffusion coefficient. The $K_{p \text {,disk }}$ factor here includes contributions from $k={ }^{12} \mathrm{C},{ }^{16} \mathrm{O}$, and ${ }^{14} \mathrm{~N}$ (even though the last one is unimportant, with $\mathrm{N} / \mathrm{O}=0.14$, compared to $\mathrm{C} / \mathrm{O}=0.5$; Lodders 2003) and $i={ }^{1} \mathrm{H},{ }^{4} \mathrm{He}$. In addition, it includes contributions from indirect reactions (Desch et al. 2004), i.e. those where the heavy nuclei originate from the cosmic rays instead of the target, since, although the hereby produced ${ }^{10} \mathrm{Be}$ will retain the momentum of the incoming particles, it should be stopped further downstream in the disk. Since Galilean invariance dictates $\sigma_{i, k}=\sigma_{k, i}$, this amounts to multiplying each "direct" reaction contribution in the right-hand side of Eq. (10) by $1+\left[(k / i)_{\mathrm{CR}} /(k / i)_{\mathrm{disk}}\right] \Sigma_{p, k}(E) / \Sigma_{p, i}(E)=1+A_{\mathrm{k}} / Z_{\mathrm{k}}^{2}$, where the second expression uses the assumption that the disk has a (solar) composition, identical to that of the cosmic rays (see also Appendix B). The resulting $K_{p \text {,disk }}$ is plotted as the "solar target" curve in Fig. 2.

Another "indirect" contribution would be solar wind implantation of ${ }^{10} \mathrm{Be}$ produced on the proto-Sun (Bricker \& Caffee 2010). Although Bricker \& Caffee (2010) originally envisioned implantation on bare solids, one can equally envision implantation on the gas disk surface (followed by vertical mixing); this would amount to adding $\left(L_{10} \mathrm{Be} / 2 \pi R\right) \partial f_{\mathrm{SW}} / \partial R$ to the right-hand side of Eq. (14), with $L^{10} \mathrm{Be}$ the young Sun's ${ }^{10} \mathrm{Be}$ (number) production rate and $f_{\mathrm{SW}}$ the counterpart of $f$. This would amount to an effective increase of $K_{p, \text { disk }}$ of $\left(\mathrm{d} f_{\mathrm{SW}} / \mathrm{d} f\right) L^{10} \mathrm{Be} /\left(L_{10} c_{\mathrm{O}}\right)$. If I set $\mathrm{d} f_{\mathrm{SW}} / \mathrm{d} f\left(L_{10} \mathrm{Be} / L_{10}\right)$ equal to the present-day ratio of long-term average $1 \mathrm{AU}{ }^{10} \mathrm{Be}(\text { Nishiizumi \& Caffee } 2001)^{3}$ and $>10 \mathrm{MeV}$ proton (Reedy 1996) energy omnidirectional fluxes, this evaluates to $6 \times 10^{-20} \mathrm{~s}^{2} \mathrm{~m}^{-2}$. From Fig. 2, at face value, solar wind implantation thus appears negligible compared to local (in-disk) spallation (except for steep $p \gtrsim 4$ ), but since we do not really know how to extrapolate ${ }^{10} \mathrm{Be}$ production on the Sun to its very active early times, the nominal one order-of-magnitude deficit may not warrant definitive conclusions yet.

Returning to Eq. (14), the requirement that ${ }^{10} \mathrm{Be} /{ }^{9} \mathrm{Be}$ vanishes at infinity leads to the first integration

$$
\left(\frac{\partial}{\partial R}-\frac{u_{\mathrm{R}}}{D_{\mathrm{R}}}\right)\left(\frac{{ }^{10} \mathrm{Be}}{{ }^{9} \mathrm{Be}}\right)_{\text {disk }}=\frac{K_{p, \text { disk }} L_{10}}{2 \pi R \Sigma D_{\mathrm{R}}}\left(\frac{\mathrm{O}}{\mathrm{Be}}\right)_{\text {disk }} f(R,+\infty) .
$$

Provided the radial Schmidt number $\mathrm{Sc}_{\mathrm{R}} \equiv v / D_{\mathrm{R}}$ has a finite lower bound, the requirement that ${ }^{10} \mathrm{Be} /{ }^{9} \mathrm{Be}$ does not diverge at the inner edge of the disk (since $u_{\mathrm{R}} / D_{\mathrm{R}}$ would not be integrable there from Eq. (3)) leads to the unique solution

$$
\begin{aligned}
\left(\frac{{ }^{10} \mathrm{Be}}{{ }^{9} \mathrm{Be}}\right)_{\text {disk }}= & K_{p, \text { disk }} L_{10}\left(\frac{\mathrm{O}}{\mathrm{Be}}\right)_{\text {disk }}\left[\frac{f(R,+\infty)}{\dot{M}}\right. \\
& \left.-\int_{R_{0}}^{R} \exp \left(\int_{R^{\prime}}^{R} \frac{u_{\mathrm{R}}}{D_{\mathrm{R}}} \mathrm{d} R^{\prime \prime}\right) \frac{\partial}{\partial R}\left(\frac{f\left(R^{\prime},+\infty\right)}{\dot{M}}\right) \mathrm{d} R^{\prime}\right] \\
= & \frac{K_{p, \text { disk }} L_{10}}{\dot{M}}\left(\frac{\mathrm{O}}{\mathrm{Be}}\right)_{\text {disk }} f_{\text {eff }},
\end{aligned}
$$

where the second equality assumes uniform $\dot{M}$ and $\mathrm{Sc}_{\mathrm{R}}$, with

$f_{\mathrm{eff}} \equiv f(R,+\infty)+\int_{R_{0}}^{R}\left(\frac{R^{\prime 1 / 2}-R_{0}^{1 / 2}}{R^{1 / 2}-R_{0}^{1 / 2}}\right)^{3 \mathrm{Sc}_{\mathrm{R}}} \frac{\partial f\left(R_{0}, R^{\prime}\right)}{\partial R} \mathrm{~d} R^{\prime}$,

which is plotted in Fig. 3 .

Interestingly, the result is weakly sensitive to the details of the protoplanetary disk (e.g., turbulence level) with the reference to heliocentric distance being only implicit in $f_{\text {eff }}$. This is because the approximate $\propto R^{-2}$ decrease of the flux density is essentially compensated by the $R^{2}$ factor in the radial transport timescale $t_{\text {vis. }}$. The above expression can be interpreted as a sum of contributions advected from outer regions (see next subsection, in particular Eq. (19)) and contributions diffused back outward. ${ }^{10} \mathrm{Be} /{ }^{9} \mathrm{Be}$ decreases monotonically outward, falling off roughly as $R^{-3 \mathrm{Sc}_{\mathrm{R}} / 2}$ outside the irradiated region, conforming to the equilibrium gradient of a passive scalar (Clarke \& Pringle 1988; Jacquet \& Robert 2013). In the limit $\mathrm{Sc}_{\mathrm{R}} \rightarrow 0$, it converges pointwise toward the inner edge value $f\left(R_{0},+\infty\right)$ and a flat profile, but $\mathrm{Sc}_{R}$ is probably of order unity in magnetohydrodynamical turbulence (Johansen et al. 2006), not to mention the possibility of laminar wind-driven accretion (and thus even higher $\mathrm{Sc}_{\mathrm{R}}$ ) further out (e.g., Bai 2016). So contrary to earlier statements (Gounelle et al. 2013; Kööp et al. 2018a), ${ }^{10} \mathrm{Be}$ production in the gas would entail no spatial uniformity of the ${ }^{10} \mathrm{Be} /{ }^{9} \mathrm{Be}$ ratio.

3 Taking into account the factor of four between the mean flux of ${ }^{10} \mathrm{Be}$ on a randomly oriented surface $\left((2.9 \pm 1.2) \times 10^{-2} \mathrm{~m}^{-2} \mathrm{~s}^{-1}\right.$ on the Moon $)$ and its omnidirectional flux in vacuo. 


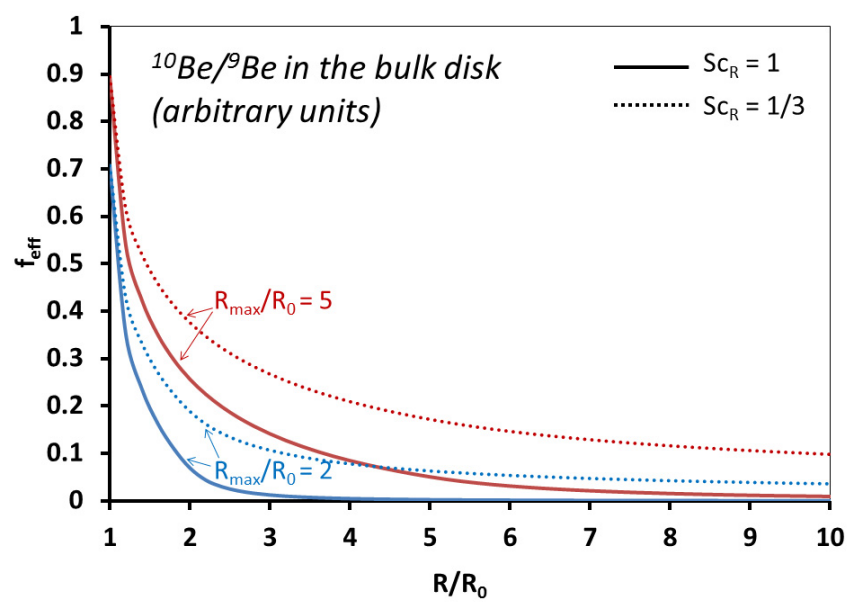

Fig. 3. $f_{\text {eff }}$ (Eq. (17)) as a function of heliocentric distances for two values of $R_{\max }$ for my toy parameterization of the particle flux distribution (see end of Sect. 2.2) and two values of the radial Schmidt number, with the lower one (higher relative diffusivity) in dotted lines. The parameter $f_{\text {eff }}$ is proportional to the ${ }^{10} \mathrm{Be} /{ }^{9} \mathrm{Be}$ ratio (whose absolute magnitude is discussed at Eq. (22)) in the bulk (gas+solids) disk for given mass accretion rate and proton output and energy distributions.

\subsection{In situ ${ }^{10} \mathrm{Be}$ production in free-floating solids}

I now consider a (sub-)millimeter rocky solid, say a CAI, formed (or more precisely, whose beryllium last equilibrated with the gas) at an heliocentric distance $R_{1}$. It will certainly inherit the ${ }^{10} \mathrm{Be} /{ }^{9} \mathrm{Be}$ ratio of the local reservoir calculated above, but it should also acquire additional ${ }^{10} \mathrm{Be}$ so long as it wanders through the irradiated region of the disk. The purpose of this subsection is to estimate this contribution.

Since the inner disk (where irradiation may occur) is dense, I assume the solid to be tightly coupled to the gas (that is, a gasgrain decoupling parameter $S \ll 1$; Jacquet et al. 2012; this is consistent with the lack of bulk chemical fractionation assumed previously). It is also small enough for the thin target approximation (e.g., Sossi et al. 2017) to apply, that is, for Eq. (5) to apply at the scale of the whole particle as a function of the local (outside) monoenergetic intensities. Since the radial transport timescale $t_{\mathrm{vis}}$ is much longer than the vertical transport timescale $H^{2} / v$ with $H=c_{\mathrm{S}} / \Omega_{\mathrm{K}}$ the pressure scale height, I can use the average rate of ${ }^{10} \mathrm{Be}$ production given by Eq. (9). That is, I view the random vertical motion of the solids due to turbulence as an ergodic process (see, e.g., Fig. 8 in Ciesla 2010) and identify the time average of this rate for a given solid to the instantaneous average over the long-term probability distribution of same-size solids, which here follows the distribution of the gas (but see Appendix $\mathrm{C}$ for the case of finite settling) ${ }^{4}$. For this refractory target, the sum on the right-hand side of Eq. (10) is essentially restricted to $k=\mathrm{O}$ and $i={ }^{1} \mathrm{H},{ }^{4} \mathrm{He}$ (with no "indirect reaction"

4 It may also be noted that since the portion of $t_{\mathrm{vis}}$ spent within a column $\Sigma_{\text {spall }}$ of the surface

$$
\begin{aligned}
t_{\text {vis }} \frac{2 \Sigma_{\text {spall }}}{\Sigma} & =\frac{6 \pi R^{2} \Sigma_{\text {spall }}}{\dot{M}\left(1-\sqrt{R_{0} / R}\right)} \\
& =\frac{5 a}{1-\sqrt{R_{0} / R}}\left(\frac{R}{0.5 \mathrm{AU}}\right)^{2}\left(\frac{10^{-7} M_{\odot} / a}{\dot{M}}\right)\left(\frac{\Sigma_{\text {spall }}}{10 \mathrm{~kg} \mathrm{~m}^{-2}}\right)
\end{aligned}
$$

is much longer than the weekly periodicity of protostellar flares (Wolk et al. 2005), the relevant proton luminosity $L_{10}$ in Eq. (9) must be the long-term average luminosity rather than the "characteristic" baseline or the typical flare peak value.

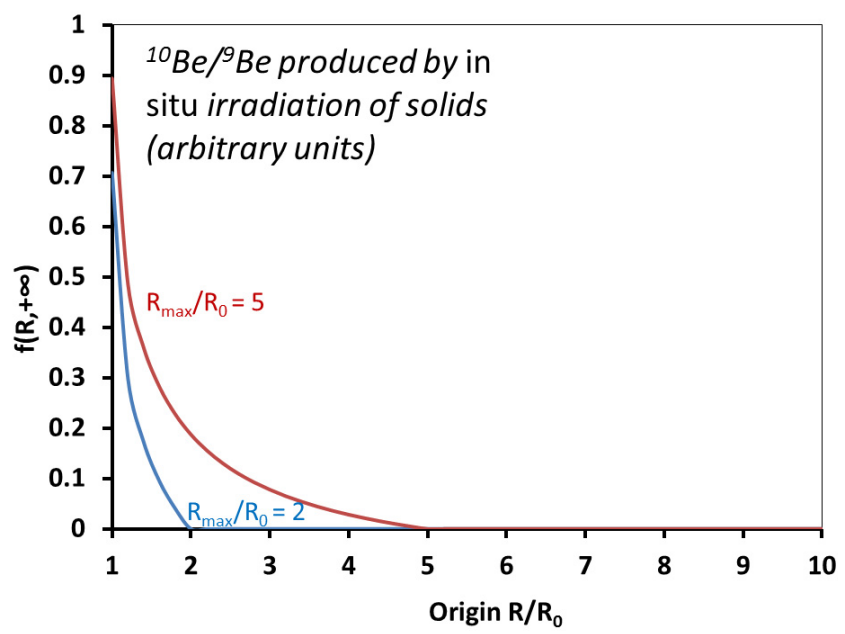

Fig. 4. $f(R,+\infty)$ as a function of heliocentric distances for two values of $R_{\max }$ for my toy parameterization of the particle flux distribution (see end of Sect. 2.2). This is proportional to the ${ }^{10} \mathrm{Be} /{ }^{9} \mathrm{Be}$ ratio produced by in situ irradiation of a refractory solid as a function of its heliocentric distance of origin.

contribution). This corresponds to the "rocky target" curve in Fig. 2.

As a result of the turbulent diffusion superimposed on advection by the gas mean velocity, the radial motion of an individual solid has a stochastic character. I will be content to set the "typical timescale" spent crossing a radial bin of width $\Delta R \ll R$ to $\left|\Delta R / u_{\mathrm{R}}\right|$. Indeed, even when the flow is opposite to the transport envisioned (as in this subsection), the fraction of a population of solids at $R$ at $t=0$, which has diffused upstream beyond that distance at time $t\left(\sim \operatorname{erfc}\left(\left(\Delta R-u_{\mathrm{R}} t\right) / \sqrt{4 D_{\mathrm{R}} t}\right) / 2\right.$, with erfc the complementary error function), is maximum for $t=-\Delta R / u_{\mathrm{R}}$. The total ${ }^{10} \mathrm{Be}$ produced in the solid during transport between an heliocentric distance $R_{1}$ and its exit from the irradiated region is then given by

$$
\begin{aligned}
\left(\frac{{ }^{10} \mathrm{Be}}{{ }^{9} \mathrm{Be}}\right)_{\mathrm{CAI}} & =\int_{R_{1}}^{+\infty}\left\langle\left(\frac{{ }^{10} \mathrm{Be}}{{ }^{9} \mathrm{Be}}\right) \sum_{\mathrm{tg}} \frac{\mathrm{d} R}{\left|u_{\mathrm{R}}\right|}\right. \\
& =K_{p, \mathrm{CAI}} L_{10}\left(\frac{\mathrm{O}}{\mathrm{Be}}\right)_{\mathrm{CAI}} \int_{R_{1}}^{+\infty} \frac{\partial f\left(R_{0}, R\right)}{\partial R} \frac{\mathrm{d} R}{\dot{M}} \\
& =\frac{K_{p, \mathrm{CAI}} L_{10}}{\dot{M}}\left(\frac{\mathrm{O}}{\mathrm{Be}}\right)_{\mathrm{CAI}} f\left(R_{1},+\infty\right),
\end{aligned}
$$

where the last equality assumes a uniform $\dot{M}(R)$. Figure 4 plots $f(R,+\infty)$. Unlike the inherited disk value (which benefited from outward diffusion), nonzero values do not extend to source radii outside the irradiated region.

\section{Discussion}

\subsection{Magnitude of ${ }^{10} \mathrm{Be}$ production}

Provided a given CAI underwent isotopic equilibration after the last production of ${ }^{10} \mathrm{Be}$, the ${ }^{10} \mathrm{Be} /{ }^{9} \mathrm{Be}$ indicated by the slope of an internal isochron is the sum of that inherited from the disk gas and that produced in situ in the solid (Eqs. (16) and (19), respectively), which may be written as

$\left(\frac{{ }^{10} \mathrm{Be}}{{ }^{9} \mathrm{Be}}\right)_{\text {final }}=\frac{K_{\mathrm{p}, \text { disk }} L_{10} f_{\text {sum }}}{\dot{M}}\left(\frac{\mathrm{O}}{\mathrm{Be}}\right)_{\text {disk }}$ 
with

$$
\begin{aligned}
f_{\text {sum }} \equiv & f\left(R_{1},+\infty\right)\left(1+\frac{K_{\mathrm{p}, \mathrm{CAI}}(\mathrm{O} / \mathrm{Be})_{\mathrm{CAI}}}{K_{\mathrm{p}, \mathrm{disk}}(\mathrm{O} / \mathrm{Be})_{\mathrm{disk}}}\right) \\
& +\int_{R_{0}}^{R_{1}}\left(\frac{R^{\prime 1 / 2}-R_{0}^{1 / 2}}{R_{1}^{1 / 2}-R_{0}^{1 / 2}}\right)^{3 \mathrm{Sc}_{R}} \frac{\partial f\left(R_{0}, R\right)}{\partial R} \mathrm{~d} R^{\prime} .
\end{aligned}
$$

The contribution of in situ production (the term with the "CAI" subscripts in the above equation) is subdominant with respect to spallation in the solar gas (that is, $\left.f_{\text {sum }} \approx f_{\mathrm{eff}}\left(R_{1}\right)\right)$ since, not to mention the ignored incipient settling (see Appendix C), (i) $K_{\text {p,disk }}$ outweighs $K_{\text {p,CAI }}$ by a factor $\sim 3$ (Fig. 2) as it includes additional production channels (e.g., target ${ }^{12} \mathrm{C}$, and indirect reactions) and (ii) $\mathrm{Be}$, as a refractory element (halfcondensation temperature of $1452 \mathrm{~K}$ according to Lodders 2003), should be concentrated relative to $\mathrm{O}$ in a CAI with respect to the overall disk. Indeed Be concentrations typically are of order 0.1 $1 \mathrm{ppm}$ in the data of McKeegan et al. (2000) and Gounelle et al. (2013), compared to the CI chondrite value of $25.2 \mathrm{ppb}$ (the latter amounting to $\mathrm{O} / \mathrm{Be}=10^{7} \approx$ half the solar value; Palme et al. 2014). Nevertheless, the fine-grained CAIs of Sossi et al. (2017) exhibit concentrations down to $6 \mathrm{ppb}^{5}$. So, at least for some Be-poor CAIs having spent a random walk in the irradiated region of the disk longer than the "typical timescale" used in Sect. 3.2, in situ production may not be entirely negligible. The deviation of the initial boron isotopic composition from solar in some CAIs, too large for an irradiated solar gas, may be a collateral effect of such a contribution (Liu et al. 2010; Gounelle et al. 2013); the same applies for cosmogenic ${ }^{3} \mathrm{He}$ and ${ }^{21} \mathrm{Ne}$ excesses found by Kööp et al. (2018a) in the same type of inclusions. This indicates that, at least for some objects, heliocentric distance $R_{1}$ was inside the proton-irradiated region of the disk.

In order to numerically evaluate Eq. (20), I will, as previous authors (Lee et al. 1998; Gounelle et al. 2001; Sossi et al. 2017), use the X-ray luminosity $L_{\mathrm{X}}$ as a proxy for $L_{10}$, since only the former can be measured from distant young stellar objects (although Ceccarelli et al. (2014) estimated a proton output equivalent to $L_{10} \gtrsim 10^{27} \mathrm{~W}$, for $L_{\mathrm{X}}<$ $7 \times 10^{24} \mathrm{~W}$, from the ionization level seen toward the single source OMC-2 FIR 4). Since their luminosities are higher than the most powerful flares of the contemporaneous Sun (e.g., Feigelson et al. 2002), solar flares are the least improper analogs in that calibration. Lee et al. (1998) derived a scaling $L_{10}=0.09 L_{\mathrm{X}}$ by ratioing the total proton and X-ray fluences of the impulsive flares of solar cycle 21 (peaking around 1980). However, while impulsive flares correlate best with X-ray outputs (Lee et al. 1998), gradual flares actually dominate the proton fluences at 1 AU (Desai \& Giacalone 2016), whatever the ratio at the X point considered by Lee et al. (1998) may be. This warrants re-examination of the scaling between X-rays and solar energetic particles (SEP) without restricting to impulsive flares. Emslie et al. (2012) evaluated the energy outputs of 38 solar eruptive events between 2002 and 2006. Those 21 with reported SEP outputs totaled a SEP output of $1.5 \times 10^{25} \mathrm{~J}$ (dominated by $>10 \mathrm{MeV}$ protons since the energy spectra is shallower below this; Mewaldt 2006) and $2.6 \times 10^{24} \mathrm{~J}$ in X-rays, hence a ratio of average $L_{10} / L_{X} \approx 6$ which $I$ adopt as a normalizing

\footnotetext{
5 It may nonetheless be wondered whether this average of SIMS point does not underestimate the bulk concentrations given the incompatible behavior of Be in melilite (Paque et al. 2014).
}

value $^{6}$. Another relevant - if more indirectly for our purpose dataset is the catalog of 314 SEP events between 1984 and 2013 of Papaioannou et al. (2016). They represent a total $>10 \mathrm{MeV}$ proton $1 \mathrm{AU}$ energy fluence of $5.1 \times 10^{3} \mathrm{~J} \mathrm{~m}^{-2}$ and total X-ray fluence of $54 \mathrm{~J} \mathrm{~m}^{-2}$ hence $\left(L_{10} / L_{X}\right) \partial f / \partial \ln R / \sin \phi \approx 23$ (which averages different geometries of the flare sources vis-à-vis the Earth) assuming isotropic distribution of SEP at 1 AU, suggestive of the same order of magnitude for $L_{10} / L_{X}$. Equation (20) then becomes

$$
\begin{aligned}
\left(\frac{{ }^{10} \mathrm{Be}}{{ }^{9} \mathrm{Be}}\right)_{\text {final }}= & 6 \times 10^{-4}\left(\frac{K_{p, \text { disk }}}{9 \times 10^{-19} \mathrm{~s}^{2} \mathrm{~m}^{-2}}\right)\left(\frac{(\mathrm{O} / \mathrm{Be})_{\text {disk }}}{2.2 \times 10^{7}}\right)\left(\frac{f_{\text {sum }}}{0.1}\right) \\
& \times\left(\frac{L_{10} / L_{X}}{6}\right)\left(\frac{L_{X}}{3 \times 10^{23} \mathrm{~W}}\right)\left(\frac{10^{-7} M_{\odot} / a}{\dot{M}}\right) .
\end{aligned}
$$

The nominal value obligingly coincides with the average one measured for regular CAIs in CV chondrites (Davis \& McKeegan 2014). This, however, should not hide the considerable uncertainties in several factors, in addition to the $L_{10} / L_{X}$ discussed above. In particular, $K_{p \text {, disk }}$, normalized to the value for $p=2.5$ typical of gradual flares favored by Sossi et al. (2017), may lose one order of magnitude or so (depending on the possible implantation contributions) for a steeper energy distribution; on the other hand, the normalization value for $f_{\text {sum }}$, inspired from the case of ballistic emission, could be an underestimate if the cosmic rays are focused toward the disk (see Fig. 3), but it depends on the essentially unknown magnetic field configuration and turbulent diffusion efficiency. While the predictive value of the model should not thus be overrated, this result does show that, in the current state of our knowledge, the evidence of extinct ${ }^{10} \mathrm{Be}$ in refractory inclusions does not mandate an origin at the very inner edge of the disk, and formation over a wider range of heliocentric distance, say $0.1-1 \mathrm{AU}$, can be envisioned. This is the main point of this work.

\subsection{Spatio-temporal variations of the ${ }^{10} \mathrm{Be} /{ }^{9} \mathrm{Be}$ ratio}

In this model, ${ }^{10} \mathrm{Be} /{ }^{9} \mathrm{Be}$ is proportional to the $L_{\mathrm{X}} / \dot{M}$ that is an observable. Since $L_{\mathrm{X}}$ has only a shallow dependence on time (e.g., $\propto t^{-0.36}$ for one solar mass according to Telleschi et al. 2007), compared to the decrease of the accretion rate $\left(\propto t^{-1.4}\right.$ according to Hartmann et al. 1998), $L_{\mathrm{X}} / \dot{M}$ should increase over time (as $t^{1.04}$ if I combine these examples although derived from different data sources; see Fig. 5). It may indeed be verified in Fig. 6 that $L_{\mathrm{X}} / \dot{M}$ anticorrelates with $\dot{M}$, if with a fair amount of scatter, which reminds us of the elusive determinants of $L_{\mathrm{X}}$. Since with decreasing $\dot{M}$ isotherms should recede toward the Sun (see Eq. (4)), $f_{\text {sum }}$ may also be expected to increase for a given $T\left(R_{1}\right)$ (e.g., a condensation front ) for a fixed magnetic field configuration. So, barring a strong decrease of $L_{10} / L_{X},{ }^{10} \mathrm{Be} /{ }^{9} \mathrm{Be}$ should increase with time for a given formation and/or equilibration temperature. That is, mass loss from the disk would increase the relative importance of the surficial layers prone to spallation, overcoming the decline in proton luminosity.

This expectation would provide an explanation for the lower ${ }^{10} \mathrm{Be} /{ }^{9} \mathrm{Be}$ of FUN CAIs in CV chondrites (MacPherson et al. 2003; Wielandt et al. 2012) and PLACs in CM chondrites (Liu et al. 2010) compared to "regular" CAIs. Indeed these CAIs are characterized by nucleosynthetic stable isotopic anomalies (e.g., in Ti, Ca; MacPherson 2014; Kööp et al. 2018b) much in excess

6 Of course, this may shorten the timescales required by Sossi et al. (2017) at the disk inner edge as well, but they would still represent dozens of orbits. 


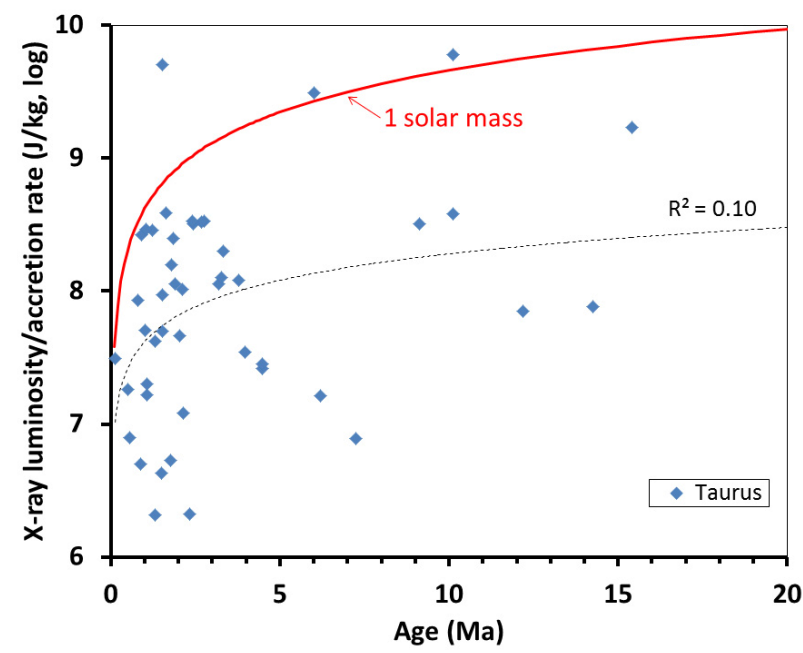

Fig. 5. $L_{\mathrm{X}} / \dot{M}$ for Taurus as a function of age (data from Güdel et al. 2007). Although a least-square fit (dashed line) shows a systematic increase with age, scatter is evident, in part owing to the diversity of stellar masses. Overplotted is the curve (red, continuous) corresponding to one solar mass (Telleschi et al. 2007; see beginning of Sect. 4.2). For reference, the normalizations used in Eq. (22) amount to $L_{\mathrm{X}} / \dot{M}=10^{7.7} \mathrm{~J} \mathrm{~kg}^{-1}$.

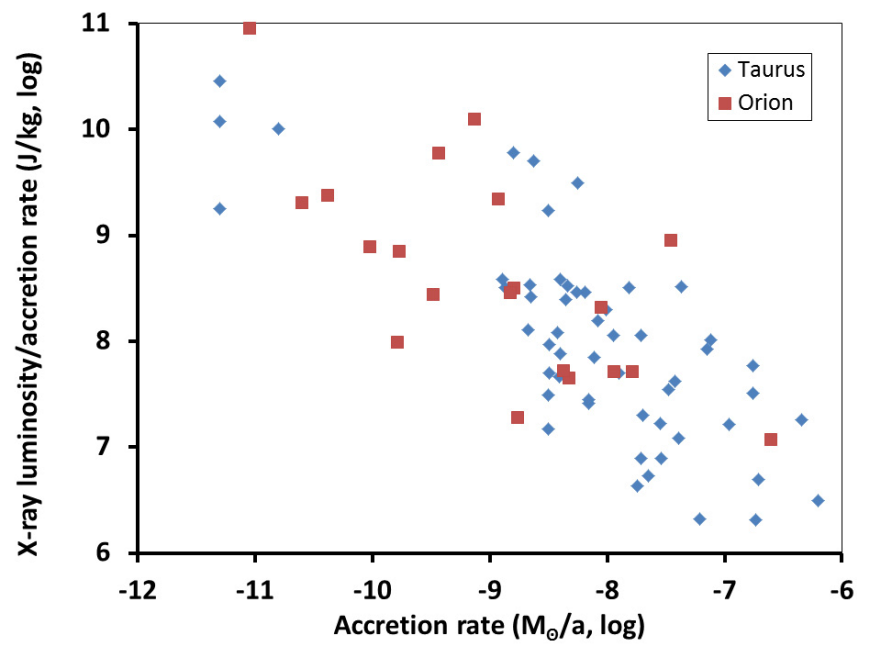

Fig. 6. $L_{\mathrm{X}} / \dot{M}$ for young stellar objects as a function of $\dot{M}$. Taurus and Orion data are taken from Güdel et al. (2007) and Bustamante et al. (2016), respectively (where $L_{X} / \dot{M}$ also shows little systematic trend with stellar mass).

of those seen in bulk chondrules (Gerber et al. 2017) or meteorites (Trinquier et al. 2009). This suggests they formed early, perhaps during infall of an isotopically heterogeneous protosolar cloud, before turbulent mixing essentially suppressed the heterogeneities (e.g., Boss 2012). Perhaps the $L_{\mathrm{X}} / \dot{M}$ was so low that their ${ }^{10} \mathrm{Be} /{ }^{9} \mathrm{Be}$ was in fact dominated by the background inherited from the protosolar cloud (with perhaps some irradiation from the protostar directly on the envelope before arrival on the disk; Ceccarelli et al. 2014) rather than later dominating local spallation (Liu et al. 2010; Wielandt et al. 2012).

At the other end of the spectrum, a relatively late formation might explain the high ${ }^{10} \mathrm{Be} /{ }^{9} \mathrm{Be}\left(7 \times 10^{-3}\right)$ of fine-grained $\mathrm{CV}$ chondrite CAIs analyzed by Sossi et al. (2017) or that of Isheyevo CAI 411 (10 ${ }^{-2}$; Gounelle et al. 2013). Indeed the former CAIs have group II rare earth element patterns that are commonly ascribed to condensation in a reservoir previously depleted in an ultrarefractory condensate (e.g., Boynton 1989). One may then speculate that this preliminary fractionation took some time to complete (given the tight coupling of millimeter-sized solids and gas) so group II CAIs had to be of relatively late formation. However, while Isheyevo CAI 411 shows ${ }^{26} \mathrm{Al} /{ }^{27} \mathrm{Al}<10^{-6}$ an ${ }^{26} \mathrm{Al}$ depletion common to many $\mathrm{CH}$ chondrite CAIs -, the fine-grained $\mathrm{CV}$ chondrite $\mathrm{CAIs}$ tend to exhibit ${ }^{26} \mathrm{Al} /{ }^{27} \mathrm{Al}$ ratios no lower than their melted counterparts (MacPherson et al. 2012; Kawasaki et al. 2019), unlike what a later formation would suggest in view of the ${ }^{26} \mathrm{Al}$ half-life of $0.72 \mathrm{Ma}$ (e.g., MacPherson et al. 2012). This conclusion is, however, predicated on an homogeneous ${ }^{26} \mathrm{Al}$ distribution, which is far from a foregone assumption in early times. In fact, the nucleosynthetic anomaly-bearing CAIs discussed above show reduced ${ }^{26} \mathrm{Al} /{ }^{27} \mathrm{Al}$ $\left(\sim 10^{-5}\right.$; MacPherson et al. 2014; Park et al. 2017) indicating an increase of that ratio in the CAI-forming region between their formation epoch and that of melted CAIs, and perhaps somewhat beyond. Interestingly also, some ${ }^{26} \mathrm{Al}$ should be produced by spallation along with ${ }^{10} \mathrm{Be}$; for their fine-grained CAIs, Sossi et al. (2017) estimated an increase of ${ }^{26} \mathrm{Al} /{ }^{27} \mathrm{Al}$ of $(0.3-$ $1.2) \times 10^{-5}$, comparable to the spread resolved by MacPherson et al. (2012) and Kawasaki et al. (2019). At any rate, this calls into question the usefulness of ${ }^{26} \mathrm{Al}$ as a chronometer during CAI formation, although it may have become more homogenized afterward. Alternatively, the coarse-grained CAIs may have acquired ${ }^{10} \mathrm{Be} /{ }^{9} \mathrm{Be}$ lower than their fine-grained counterparts because of equilibration with their surroundings at higher heliocentric distances, during the localized heating events that melted them.

This very dependence of ${ }^{10} \mathrm{Be} /{ }^{9} \mathrm{Be}$ on heliocentric distance also implies that the comparatively younger chondrules (or other meteoritic material), which likely formed several AUs away from the Sun (so probably outside the range for in situ irradiation), need not have high ${ }^{10} \mathrm{Be} /{ }^{9} \mathrm{Be}$, not to mention effects of finite time of transport or radioactive decay, as timescales for the chondrule formation epoch (0-3 Ma after CAIs; Nagashima et al. 2018; Connelly \& Bizzarro 2018) or their transport are comparable to the half-life of ${ }^{10} \mathrm{Be}$. For a Schmidt number of order unity, one might expect a $2 \pm 1$ orders of magnitude depletion with respect to the CAI-forming region (one order of magnitude closer to the Sun), but it is difficult to narrow down. Still, no chondrule ${ }^{10} \mathrm{Be} /{ }^{9} \mathrm{Be}$ values are known yet since silicate crystallization there hardly fractionates Be from B (Davis \& McKeegan 2014).

\subsection{Implications for vanadium isotopic systematics}

While it is beyond the scope of the current work to calculate the effects of proton irradiation on other isotopic systems, some comments on the reinterpretation of the vanadium-50 excesses (up to 4.4\%o) reported by Sossi et al. (2017) are in order. In this framework, spallogenic ${ }^{50} \mathrm{~V}$ would also be dominated by inheritance from the nebula, rather than in situ production, but to a lesser extent as the target nuclei/element ratio (essentially $\mathrm{Ti} / \mathrm{V}$ playing the role of O/Be; Sossi et al. 2017), between two refractory elements, would not markedly differ between a solar gas and a condensate (see second paragraph of Sect. 4.1). So the contention by Kööp et al. (2018a) that lack of evidence of in situ production of noble gases by solar energetic production in fine-grained CAIs analyzed by Vogel et al. (2004) ${ }^{7}$ prevents

\footnotetext{
7 These incidentally do show evidence for spallogenic $\mathrm{He}$ and $\mathrm{Ne}$, even though it is unclear there is any excess over that due to the recent exposure of the meteoroid to galactic cosmic rays.
} 
a spallogenic origin for their ${ }^{50} \mathrm{~V}$ excesses and favors massdependent fractionation upon condensation is not necessarily valid. Since differential attenuation of the energetic particles modifies their energy distribution at finite penetration columns, the production ratio (independent of the fluence, and only dependent on $p$ ) of two nuclides would be affected. In particular, for a given $p$, since the reactions producing ${ }^{50} \mathrm{~V}$ are efficient at lower energies than for ${ }^{10} \mathrm{Be}$ (Lee et al. 1998), where attenuation is strongest, ${ }^{50} \mathrm{~V}$ production would be comparatively lower. Thus, for given (measured) ${ }^{10} \mathrm{Be} /{ }^{9} \mathrm{Be}$ and $\delta^{51} \mathrm{~V}$, a steeper slope than in the formalism of Sossi et al. (2017) would be indicated (for a given target composition, which however would have to be revised to solar). Since, if we ratio Eq. (8) with its ${ }^{50} \mathrm{~V}$ counterpart, the only difference with the Sossi et al. (2017) formalism is the presence of $\Sigma_{p}(E)$ in the integrals in the denominator and the numerator, one may surmise that a shift of $p$ comparable to the power-law exponent of $\Sigma_{p}(E)$ in the Continuous Slowing Down Approximation (about 1.82; see Appendix B) would largely cancel out its effect and restore the observed ratio. Although a steeper $p$ would diminish ${ }^{10} \mathrm{Be}$ production for a given $L_{10}$, this would hardly affect our conclusions given the other uncertainties discussed at the end of Sect. 4.1. This also does not take into account the neutron contribution alluded to in Sect. 2.2, which may alter inferences on the energy distribution. Clearly, a dedicated study on the simultaneous effects expected for different isotopic systems in this scenario, whose proportions are independent of the uncertainties on the fluences, would be worthwhile.

\section{4. $X$-rays and $D / H$ fractionation}

As mentioned above, X-ray emission should accompany cosmic ray flares from the Sun. These could also leave isotopic fingerprints (though unrelated to spallation) in meteorites. Indeed Gavilan et al. (2017) linked deuterium enrichment of chondritic organic matter (previously ascribed by Remusat et al. 2006, 2009 to ionizing radiation) to X-ray irradiation near the surface of the disk. The energy fluence $F$ expected for organic matter wandering in the outer disk can be calculated with the same formalism as above if I replace $\sigma_{k, i}(E)(i / \mathrm{H})_{\mathrm{CR}}(k / \mathrm{Be})_{\mathrm{tg}}$ by $E$, so I end this discussion with this short aside. For an attenuation column $\Sigma_{\mathrm{X}}(E)=0.15 \mathrm{~kg} \mathrm{~m}^{-2}(\mathrm{E} / 1 \mathrm{keV})^{n}$ with $n=2.485$ and an energy distribution $E I_{\mathrm{X}} \propto \exp \left(-E /\left(k_{\mathrm{B}} T_{\mathrm{X}}\right)\right)$ with $T_{\mathrm{X}}$ the emission temperature (Igea \& Glassgold 1999), the energyweighted average of the former (the equivalent of $K_{p}$ ) is $\Sigma_{\mathrm{X}}\left(k_{\mathrm{B}} T_{\mathrm{X}}\right) \Gamma(n+1)$. For distant part of the (flared) disk, I can use $f\left(R_{0}, R\right)=H_{\mathrm{X}} / R$ (with $H_{\mathrm{X}}$ the X-ray absorption height, likely a few times $H$ by analogy with Appendix C) so that the net fluence has the fairly well-constrained expression (modified after Eq. (19))

$$
\begin{aligned}
F= & \Gamma(n+1) \Sigma_{\mathrm{X}}\left(k_{\mathrm{B}} T_{\mathrm{X}}\right) \frac{L_{\mathrm{X}}}{\dot{M}}\left[\frac{H_{\mathrm{X}}}{R}\right] \\
= & 2 \times 10^{7} \mathrm{~J} \mathrm{~m}^{-2}\left(\frac{\Gamma(n+1)}{3}\right)\left(\frac{\Sigma_{X}(1 \mathrm{keV})}{0.15 \mathrm{~kg} \mathrm{~m}^{-2}}\right)\left(\frac{k_{\mathrm{B}} T_{\mathrm{X}}}{1 \mathrm{keV}}\right)^{n} \\
& \times\left(\frac{L_{\mathrm{X}}}{3 \times 10^{23} \mathrm{~W}}\right)\left(\frac{10^{-8} M_{\odot} / a}{\dot{M}}\right)\left(\frac{\left[H_{\mathrm{X}} / R\right]}{0.1}\right),
\end{aligned}
$$

with $\left[H_{\mathrm{X}} / R\right]$ the difference of $H_{\mathrm{X}} / R$ between the locus of formation of the organic matter (or the location inward of which settling ceases to be important, i.e. the $S=1$ line of Jacquet et al. 2012; see Appendix C) and that of accretion in the chondrite parent body. This is five orders of magnitude short of the critical fluence of $5 \times 10^{27} \mathrm{eV} \mathrm{cm}^{-2}=8 \times 10^{12} \mathrm{~J} \mathrm{~m}^{-2}$ indicated by the experiments of Gavilan et al. (2017) for $0.5-1.3 \mathrm{keV}$ photons (and is also much shorter than the astrophysical estimate of Gavilan et al. 2017, which erroneously used X-ray attenuation at surface layers as representative of the bulk). While the steady-state approximation may overestimate the surface density in the outer regions of interest (which nevertheless should not allow efficient settling of the grains), alleviating it would hardly bridge the gap. Nevertheless, the quantitative assessment of isotopic effects of irradiation, whether X-rays (in particular at higher energies) or other parts of the spectrum, is still in its infancy and this additional calculation is essentially intended for future applications in similar scenarios.

\section{Conclusion}

I have analytically investigated the production of short-lived radionuclide beryllium-10 in surface layers of the disk irradiated by protosolar flares. I found that ${ }^{10} \mathrm{Be}$ production in the gas outweighs ${ }^{10} \mathrm{Be}$ production in solids after condensation because the gas contains a greater breadth of suitable target nuclides (e.g., ${ }^{12} \mathrm{C},{ }^{1} \mathrm{H}$ ) and incurs less dilution by stable refractory Be. Taking into account incipient settling and possible implantation of solar wind-borne ${ }^{10} \mathrm{Be}$ would further widen the difference. Although many uncertainties remain on the magnetic field configuration, the scaling of cosmic rays with X-ray luminosities and other factors, it does appear that this model can reproduce ${ }^{10} \mathrm{Be} /{ }^{9} \mathrm{Be}$ ratios measured in CAIs. Therefore, the past presence of ${ }^{10} \mathrm{Be}$ does not require (at least at present) that CAIs formed at the inner edge of the disk and allows formation at a fraction of an $\mathrm{AU}$, as thermal models would suggest, and more in line with evidence for a genetic link with their host carbonaceous chondrites (abundance, fraction of the refractory budget; Jacquet et al. 2012). If this model holds true, an interesting corollary (barring strong variations of the energetic protons/X-ray ratio) is that the oldest CAIs should have the lowest ${ }^{10} \mathrm{Be} /{ }^{9} \mathrm{Be}$ ratios, which would explain those of nucleosynthetic anomalies-bearing CAIs ((F)UN, PLAC). This would also suggest that the fine-grained group II CAIs in CV chondrites measured by Sossi et al. (2017) were a relatively late generation of refractory inclusions, a possibility that remains to be explored. This does not mean that chondrules, which formed at comparatively much larger heliocentric distances, should have high ${ }^{10} \mathrm{Be} /{ }^{9} \mathrm{Be}$, since this ratio decreases outward, following passive diffusion outside the irradiated inner disk. I finally note that the same formalism allows an estimate of fluences of X-rays (produced in the same protosolar flares) or other types of radiations, on aggregates freely floating in the disk which can be, for example, compared to experimental evidence of $\mathrm{D} / \mathrm{H}$ fractionation in meteoritic organic matter by irradiation.

Acknowledgments. I am grateful to the organizers and participants of the workshop "Core to Disk", a program of the $\psi 2$ initiative that took place at the Institut d'Astrophysique Spatiale in Orsay from May 14 to June 22, 2018, in particular Patrick Hennebelle and Matthieu Gounelle, who elicited my interest in the subject. I also thank Dr Ming-Chang Liu, Manuel Güdel, and Thomas Preibisch who kindly answered my requests for information. Comments by the anonymous reviewer greatly improved the clarity of the result sections as well as the discussion of other isotopic systems. This work is supported by ANR-15-CE31-004-1 (ANR CRADLE).

\section{References}

Bai, X.-N. 2016, ApJ, 821, 80

Balbus, S. A., \& Hawley, J. F. 1998, Rev. Mod. Phys., 70, 1

Boss, A. P. 2012, Annu. Rev. Earth Planet. Sci., 40, 23 
Boynton, W. V. 1989, in Geochemistry and Mineralogy of the Rare Earth Elements, eds. R. B. Lipin \& G. A. McKay (Chantilly: Mineralogical Society of America), 21, 1

Bricker, G. E., \& Caffee, M. W. 2010, ApJ, 725, 443

Bustamante, I., Merín, B., Bouy, H., et al. 2016, A\&A, 587, A81

Cassen, P., \& Moosman, A. 1981, Icarus, 48, 353

Ceccarelli, C., Dominik, C., López-Sepulcre, A., et al. 2014, ApJ, 790, L1

Ciesla, F. J. 2010, ApJ, 723, 514

Clarke, C. J., \& Pringle, J. E. 1988, MNRAS, 235, 365

Connelly, J. N., \& Bizzarro, M. 2018, The Absolute Pb-Pb Isotope Ages of Chondrules: Insights into the Dynamics of the Solar Protoplanetary Disk, eds. S. S. Russell, A. N. Krot, \& H. C. J. Connolly (Cambridge: Cambridge University Press, 247

Davis, A. M., \& McKeegan, K. D. 2014, Short-Lived Radionuclides and Early Solar System Chronology (Amsterdam: Elsevier), 361

Davis, A. M., \& Richter, F. M. 2014, Condensation and Evaporation of Solar System Materials (Amsterdam: Elsevier), 335

Desai, M., \& Giacalone, J. 2016, Liv. Rev. Sol. Phys., 13, 3

Desch, S. J., Connolly, Jr. H. C., \& Srinivasan, G. 2004, ApJ, 602, 528

Desch, S. J., Morris, M. A., Connolly, Jr. H. C., \& Boss, A. P. 2010, ApJ, 725, 692

Donati, J.-F., \& Landstreet, J. D. 2009, ARA\&A, 47, 333

Ebert, S., \& Bischoff, A. 2016, Geochim. Cosmochim. Acta, 177, 182

Emslie, A. G., Dennis, B. R., Shih, A. Y., et al. 2012, ApJ, 759, 71

Favre, C., López-Sepulcre, A., Ceccarelli, C., et al. 2017, A\&A, 608, A82

Favre, C., Ceccarelli, C., López-Sepulcre, A., et al. 2018, ApJ, 859, 136

Feigelson, E. D., Garmire, G. P., \& Pravdo, S. H. 2002, ApJ, 572, 335

Gavilan, L., Remusat, L., Roskosz, M., et al. 2017, ApJ, 840, 35

Gerber, S., Burkhardt, C., Budde, G., Metzler, K., \& Kleine, T. 2017, ApJ, 841, L17

Gounelle, M., Shu, F. H., Shang, H., et al. 2001, ApJ, 548, 1051

Gounelle, M., Shu, F. H., Shang, H., et al. 2006, ApJ, 640, 1163

Gounelle, M., Chaussidon, M., \& Rollion-Bard, C. 2013, ApJ, 763, L33

Grossman, L. 2010, Meteor. Planet. Sci., 45, 7

Güdel, M., Skinner, S. L., Mel'Nikov, S. Y., et al. 2007, A\&A, 468, 529

Hartmann, L., Calvet, N., Gullbring, E., \& D’Alessio, P. 1998, ApJ, 495, 385

Hayashi, C. 1981, Prog. Theor. Phys. Suppl., 70, 35

Hezel, D. C., Russell, S. S., Ross, A. J., \& Kearsley, A. T. 2008, Meteor. Planet. Sci., 43, 1879

Igea, J., \& Glassgold, A. E. 1999, ApJ, 518, 848

Jacquet, E. 2013, A\&A, 551, A75

Jacquet, E. 2014, Comptes Rendus Geoscience, 346, 3

Jacquet, E., \& Robert, F. 2013, Icarus, 223, 722

Jacquet, E., \& Marrocchi, Y. 2017, Meteor. Planet. Sci., 52, 2672

Jacquet, E., Gounelle, M., \& Fromang, S. 2012, Icarus, 220, 162

Johansen, A., Klahr, H., \& Mee, A. J. 2006, MNRAS, 370, L71

Jones, R. H., \& Schilk, A. J. 2009, Geochim. Cosmochim. Acta, 73, 5854

Kawasaki, N., Park, C., Sakamoto, N., et al. 2019, Earth Planet. Sci. Lett., 511, 25

Kööp, L., Heck, P. R., Busemann, H., et al. 2018a, Nat. Astron., 2, 709

Kööp, L., Nakashima, D., Heck, P. R., et al. 2018b, Geochim. Cosmochim. Acta, 221, 296

Krot, A., Petaev, M., Russell, S. S., et al. 2004, Chemie der Erde / Geochemistry, 64, 185

Lange, H.-J., Hahn, T. R. M., et al. 1995, Appl. Radiat. Isot., 46, 93

Lee, T., Shu, F. H., Shang, H., Glassgold, A. E., \& Rehm, K. E. 1998, ApJ, 506, 898

Leya, I., \& Masarik, J. 2009, Meteorit. Planet. Sci., 44, 1061

Liu, M.-C., McKeegan, K. D., Goswami, J. N., et al. 2009, Geochim. Cosmochim. Acta, 73, 5051
Liu, M.-C., Nittler, L. R., Alexander, C. M. O., \& Lee, T. 2010, ApJ, 719, L99

Lodders, K. 2003, ApJ, 591, 1220

Lugaro, M., Ott, U., \& Kereszturi, Á. 2018, Prog. Part. Nucl. Phys., 102, 1

MacPherson, G. J. 2014, Calcium-Aluminum-Rich Inclusions in Chondritic Meteorites, ed. A. M. Davis (Amsterdam: Elsevier), 139

MacPherson, G. J., Huss, G. R., \& Davis, A. M. 2003, Geochim. Cosmochim. Acta, 67, 3165

MacPherson, G. J., Kita, N. T., Ushikubo, T., Bullock, E. S., \& Davis, A. M. 2012, Earth Planet. Sci. Lett., 331, 43

MacPherson, G. J., Davis, A. M., \& Zinner, E. K. 2014, Lunar Planet. Sci. Conf., 45, 2134

Marrocchi, Y., Villeneuve, J., Batanova, V., Piani, L., \& Jacquet, E. 2018, Earth Planet. Sci. Lett., 496, 132

McKeegan, K. D., Chaussidon, M., \& Robert, F. 2000, Science, 289, 1334

Metzler, K., \& Pack, A. 2016, Meteor. Planet. Sci., 51, 276

Mewaldt, R. A. 2006, Space Sci. Rev., 124, 303

Mewaldt, R. A., Cohen, C. M. S., Mason, G. M., et al. 2007, Space Sci. Rev., 130, 207

Misawa, K., \& Nakamura, N. 1988, Geochim. Cosmochim. Acta, 52, 1699

Nagashima, K., Kita, N. T., \& Luu, T. H. 2018, ${ }^{26} \mathrm{Al}-{ }^{26} \mathrm{Mg}$ Systematics of Chondrules, eds. S. S. Russell, A. N. Krot, \& H. C. J. Connolly (Cambridge: Cambridge University Press), 247

Nanne, J. A. M., Nimmo, F., Cuzzi, J. N., \& Kleine, T. 2019, Earth Planet. Sci. Lett., 511, 44

Nishiizumi, K., \& Caffee, M. W. 2001, Science, 294, 352

Padovani, M., Ivlev, A. V., Galli, D., \& Caselli, P. 2018, A\&A, 614, A111

Padovani, M., Marcowith, A., Hennebelle, P., \& Ferrière, K. 2016, A\&A, 590, A8

Palme, H., Lodders, K., \& Jones, A. 2014, Solar System Abundances of the Elements, ed. A. M. Davis (Amsterdam: Elsevier), 15

Papaioannou, A., Sandberg, I., Anastasiadis, A., et al. 2016, J. Space Weather Space Clim., 6, A42

Paque, J. M., Burnett, D. S., Beckett, J. R., \& Guan, Y. 2014, Lunar and Planet. Sci. Conf., 45, 2176

Park, C., Nagashima, K., Krot, A. N., et al. 2017, Geochim. Cosmochim. Acta, 201, 6

Preibisch, T., Kim, Y.-C., Favata, F., et al. 2005, ApJS, 160, 401

Reedy, R. C. 1996, in Solar Drivers of the Interplanetary and Terrestrial Disturbances, eds. K. S. Balasubramaniam, S. L. Keil, \& R. N. Smartt, ASP Conf. Ser., 95, 429

Remusat, L., Palhol, F., Robert, F., Derenne, S., \& France-Lanord, C. 2006, Earth Planet. Sci. Lett., 243, 15

Remusat, L., Robert, F., Meibom, A., et al. 2009, ApJ, 698, 2087

Shu, F. H., Shang, H., Gounelle, M., Glassgold, A. E., \& Lee, T. 2001, ApJ, 548, 1029

Sossi, P. A., Moynier, F., Chaussidon, M., et al. 2017, Nature Astron., 1, 0055

Telleschi, A., Güdel, M., Briggs, K. R., Audard, M., \& Palla, F. 2007, A\&A, 468, 425

Trinquier, A., Elliott, T., Ulfbeck, D., et al. 2009, Science, 324, 374

Umebayashi, T., \& Nakano, T. 1981, PASJ, 33, 617

Vogel, N., Baur, H., Bischoff, A., Leya, I., \& Wieler, R. 2004, Meteorit. Planet. Sci., 39, 767

Wielandt, D., Nagashima, K., Krot, A. N., et al. 2012, ApJ, 748, L25

Wolk, S. J., Harnden, Jr. F. R., Flaccomio, E., et al. 2005, ApJS, 160, 423

Wood, J. A. 2004, Geochim. Cosmochim. Acta, 68, 4007

Wooden, D., Desch, S., Harker, D., Gail, H., \& Keller, L. 2007, Protostars and Planets V (Tucson: University of Arizona Press), 815

Yanasak, N. E., Wiedenbeck, M. E., Mewaldt, R. A., et al. 2001, ApJ, 563, 768 


\section{Appendix A: Steady-state disk with infall}

In this appendix, I study the effects of infall on the steady-state solution for the disk. I adopt the Cassen \& Moosman (1981) expression for the infall source term per unit area $\dot{\Sigma}_{\text {in }}$ for a cloud in solid-body rotation, assuming bipolar jets do not suppress it in the inner disk. The mass accretion rate is no longer constant but obeys

$-\frac{\partial \dot{M}}{\partial R}=2 \pi R \dot{\Sigma}_{\text {in }}=\frac{\dot{M}_{\text {in }}}{2 R_{\mathrm{C}} \sqrt{1-R / R_{\mathrm{C}}}} \theta\left(R_{\mathrm{C}}-R\right)$,

with $R_{\mathrm{C}}$ the instantaneous centrifugal radius, $\dot{M}_{\text {in }}$ the total infall rate on the star+disk system, and $\theta$ the Heaviside function. Treating all matter arriving inside $R=R_{0}$ as directly accreted by the star, this can be integrated as

$\dot{M}=\dot{M}_{\star}-\dot{M}_{\text {in }}\left(1-\sqrt{1-\frac{R}{R_{\mathrm{C}}}}\right)$,

with $\dot{M}_{\star}$ the mass accretion rate of the star. If the whole disk can be treated as in steady state, we should have $\dot{M}_{\star}=\dot{M}_{\text {in }}$ but I refrain from making this identification immediately as the steady-state approximation may only hold locally in the inner regions and/or in the absence of infall $\left(\dot{M}_{\text {in }}=0\right)$ altogether (as assumed in the main text).

Angular momentum conservation reads (Cassen \& Moosman 1981)

$\dot{M}=6 \pi R^{1 / 2} \frac{\partial}{\partial R}\left(R^{1 / 2} \Sigma v\right)+2 \pi R^{2} \Sigma \frac{\dot{M}_{\star}}{M_{\star}}+4 \pi R^{2} \dot{\Sigma}_{\text {in }}\left(1-\sqrt{\frac{R}{R_{\mathrm{C}}}}\right)$,

with $M_{\star}$ the stellar mass. If the latter is much bigger than the mass of the disk, the middle term on the right-hand side can be neglected upon integration (after division by $R^{1 / 2}$ ), which yields

$\left(\dot{M}_{\star}-\dot{M}_{\text {in }}\right)\left(1-\sqrt{\frac{R_{0}}{R}}\right)+\dot{M}_{\text {in }}\left(\frac{R_{\mathrm{C}}}{R}\right)^{1 / 2}\left[\sqrt{1-x}\left(x^{1 / 2}-\frac{x+2}{3}\right)\right]_{R_{0} / R_{\mathrm{C}}}^{R / R_{\mathrm{C}}}=3 \pi \Sigma \nu$.

For $R \ll R_{\mathrm{C}}$ this approximates to

$3 \pi \Sigma v \approx\left(\dot{M}_{\star}-\dot{M}_{\mathrm{in}} \frac{R+\sqrt{R R_{0}}+R_{0}}{2 R_{\mathrm{C}}}\right)\left(1-\sqrt{\frac{R_{0}}{R}}\right) \approx \dot{M}\left(1-\sqrt{\frac{R_{0}}{R}}\right)\left(1-\frac{\dot{M}_{\mathrm{in}}}{\dot{M}_{\star}} \frac{\sqrt{R R_{0}}+R_{0}}{2 R_{\mathrm{C}}}\right)$,

which is thus a modest correction to Eq. (2).

For $R \gg R_{0}$ and $\dot{M}_{\star}=\dot{M}_{\text {in }}$, I have

$\dot{M}_{\text {in }}\left[\sqrt{1-\frac{R}{R_{\mathrm{C}}}}\left(1-\frac{\left(R / R_{\mathrm{C}}\right)^{1 / 2}}{3}\right)+\frac{2}{3}\left(\frac{R_{\mathrm{C}}}{R}\right)^{1 / 2}\left(1-\sqrt{1-\frac{R}{R_{\mathrm{C}}}}\right)\right]=3 \pi \Sigma v$.

The factor between brackets decreases from 1 to $2 / 3$ when $R$ increases from 0 to $R_{\mathrm{C}}$. Beyond the centrifugal radius, $\dot{M}=0$ and, from Eq. (A.3), $R^{1 / 2} \Sigma v$ becomes constant so that

$3 \pi \Sigma v=\frac{2}{3} \dot{M}_{\text {in }}\left(\frac{R_{\mathrm{C}}}{R}\right)^{1 / 2}$.

For bounded $\alpha T, 2 \pi R \Sigma$ is not integrable, but this steady-state solution, which merely sets an upper bound to the disk viscous expansion, can only hold for distances of $t_{\mathrm{vis}}(R)$ shorter than the timescale of evolution of the system ${ }^{8}$.

\section{Appendix B: Calculation of $K_{\mathrm{p}}$}

I take the attenuation column for protons as the minimum between the continuous slowing-down approximation (CSDA) and diffusion regimes of Padovani et al. (2018), after integrating their Eqs. (E.3) and (21) ${ }^{9}$, respectively:

8 Mathematically, inclusion of the hitherto neglected middle term of the right-hand side of Eq. (A.3) would suffice to make the disk mass finite but on a too large radial scale for it to be relevant.

9 For the latter, after a change of variable $E_{0}=E / \sin \theta$, I use the identity

$\int_{0}^{\pi / 2} \sin ^{a} \theta \mathrm{d} \theta=\frac{\sqrt{\pi}}{2} \frac{\Gamma\left(\frac{a+1}{2}\right)}{\Gamma\left(\frac{a}{2}+1\right)} ;$

(for derivation, multiply the left-hand side by $\Gamma(1+a / 2)=2 \int_{0}^{+\infty} r^{a+1} \exp \left(-r^{2}\right) \mathrm{d} r$ and rewrite the resulting double integral in Cartesian coordinates). 
Table B.1. Cross-section parameters.

\begin{tabular}{ccccc}
\hline \hline Reaction & $E_{\text {th }}(\mathrm{MeV})$ & $\sigma_{0}(\mathrm{mb})$ & $E_{0}(\mathrm{MeV})$ & $x$ \\
\hline${ }^{12} \mathrm{C}(\mathrm{p}, \mathrm{x}){ }^{10} \mathrm{Be}$ & 27.4 & 3.79 & 1037 & 0.53 \\
${ }^{14} \mathrm{~N}(\mathrm{p}, \mathrm{x}){ }^{10} \mathrm{Be}$ & 32.1 & 1.75 & 671 & 0.715 \\
${ }^{16} \mathrm{O}(\mathrm{p}, \mathrm{x}){ }^{10} \mathrm{Be}$ & 34.6 & 2.52 & 798 & 0.962 \\
${ }^{12} \mathrm{C}(\alpha, \mathrm{x}){ }^{10} \mathrm{Be}$ & 19.675 & 9.38 & 34 & 1.4 \\
${ }^{14} \mathrm{~N}(\alpha, \mathrm{x}){ }^{10} \mathrm{Be}$ & 11.05 & 5.26 & 30 & 3.4 \\
${ }^{16} \mathrm{O}(\alpha, \mathrm{x}){ }^{10} \mathrm{Be}$ & 13.45 & 4.35 & 29 & 4.3 \\
\hline
\end{tabular}

$\Sigma_{p, H}(E)=m \min \left[\frac{E^{1+s}}{A(p-1)}, \frac{1}{2(p-1)} \sqrt{\frac{E\left(\alpha_{p}-1\right)}{3 \sigma_{M T} L_{p}(E)}} \frac{\Gamma\left(\frac{p-1}{\alpha_{p}-1}+1\right)}{\Gamma\left(\frac{p-1}{\alpha_{p}-1}+\frac{1}{2}\right)}\right]=\frac{1}{p-1} \min \left[1.2 \mathrm{~kg} \mathrm{~m}^{-2}\left(\frac{E}{10 \mathrm{MeV}}\right)^{1.82}\right.$,

$\left.6 \times 10^{2} \mathrm{~kg} \mathrm{~m}^{-2}\left(\frac{1 \mathrm{GeV}}{E}\right)^{0.14} \frac{\Gamma\left(\frac{p-1}{\alpha_{p}-1}+1\right)}{\Gamma\left(\frac{p-1}{\alpha_{p}-1}+\frac{1}{2}\right)}\right]$,

where $A=1.77 \times 10^{-6} \mathrm{eV}^{1+\mathrm{s}} \mathrm{m}^{2}, s=0.82, \alpha_{p}=1.28$ (noted $\alpha$ in Padovani et al. 2018), $\sigma_{M T}=10^{-30} \mathrm{~m}^{2}, L_{p}(E)=10^{-21} \mathrm{~m}^{2} \mathrm{eV}$ $(E / \mathrm{GeV})^{\alpha_{p}}$ are defined in Padovani et al. (2018) and $\Gamma$ is Euler's gamma function. For the other species, I adopt $\Sigma_{p, i}=\Sigma_{p, H} A_{i} / Z_{i}^{2}$, with $Z_{i}, A_{i}$ its atomic and mass numbers of species $i$ (e.g., Gounelle et al. 2001). The transition between the CSDA and the diffusion regime thus occurs at

$E_{\text {crit }}=0.33 \mathrm{GeV}\left(\frac{\Gamma\left(\frac{p-1}{\alpha_{p}-1}+1\right)}{\Gamma\left(\frac{p-1}{\alpha_{p}-1}+\frac{1}{2}\right)}\right)^{0.51}$.

As to the cross sections, similar to Desch et al. (2004), I adopt the Yanasak et al. (2001) formulation

$\sigma_{k, i}(E)=\sigma_{0} \min \left(\left(\frac{E}{E_{0}}\right)^{x}, 1\right) \theta(E-E$ th $)$

with $\sigma_{0}, E$ th, $E_{0}, x$ constants (for a given $(k, i)$ ) and $\theta$ is again the Heaviside function. I fit those for $i={ }^{4} \mathrm{He}$ using the Lange et al. (1995) data (unknown to Yanasak et al. 2001), with power-law fitting below $30 \mathrm{MeV}$ nucleon $^{-1}$ and the flat regime being assigned the maximum cross section measured (rather than a fit beyond the aforementioned threshold, as this regime is only incipient in the energy range studied). The resulting parameters are presented in Table B.1.

Then I have for $E$ th $\leq E_{0} \leq E_{\text {crit }}$

$\int_{0}^{+\infty} \sigma_{k, i}(E) \Sigma_{p, i} E^{-p} \mathrm{~d} E=\sigma_{0} \frac{\Sigma_{p, i}^{\mathrm{CSDA}}\left(E_{0}\right)}{E_{0}^{p-1}}\left(\frac{1-\left(E_{\mathrm{th}} / E_{0}\right)^{2+s+x-p}}{2+s+x-p}+\frac{\left(E_{\mathrm{crit}} / E_{0}\right)^{2+s-p}-1}{2+s-p}+\frac{\left(E_{\mathrm{crit}} / E_{0}\right)^{2+s-p}}{p+\left(\alpha_{p}-3\right) / 2}\right)$,

and for $E_{\text {th }} \leq E_{\text {crit }} \leq E_{0}$

$$
\begin{aligned}
\int_{0}^{+\infty} \sigma_{k, i}(E) \Sigma_{p, i} E^{-p} \mathrm{~d} E= & \sigma_{0} \frac{\sum_{p, i}^{\mathrm{CSDA}}\left(E_{0}\right)}{E_{0}^{p-1}}\left(\frac{\left(E_{\mathrm{crit}} / E_{0}\right)^{2+s+x-p}-\left(E \mathrm{th} / E_{0}\right)^{2+s+x-p}}{2+s+x-p}+\frac{\left(E_{\mathrm{crit}} / E_{0}\right)^{s+\left(\alpha_{p}+1\right) / 2}-\left(E_{\mathrm{crit}} / E_{0}\right)^{2+s+x-p}}{x-p-\left(\alpha_{p}-3\right) / 2}\right. \\
& \left.+\frac{\left(E_{\mathrm{crit}} / E_{0}\right)^{s+\left(\alpha_{p}+1\right) / 2}}{p+\left(\alpha_{p}-3\right) / 2}\right)
\end{aligned}
$$

whose contributions can be summed to yield $K_{\mathrm{p}}$ (Eq. (10)).

\section{Appendix C: Height of spallation layer and effect of settling on ${ }^{10} \mathrm{Be}$ production in solids}

The spallation layer column $\Sigma_{\text {spall }}$ may be defined as

$\Sigma_{\text {spall }} \equiv \frac{1}{\left({ }^{10} \mathrm{Be} /{ }^{9} \mathrm{Be}\right)_{\mathrm{tg},+}(0)} \int_{0}^{+\infty}\left(\frac{{ }^{10} \mathrm{Be}}{{ }^{9} \mathrm{Be}}\right)_{\mathrm{tg},+} \mathrm{d} \Sigma^{\prime}=\left\langle\Sigma_{p}(E)\right\rangle_{\sigma E^{-p}} \frac{\sin \phi}{2}$. 
Assuming a vertically isothermal disk (and thus a gaussian gas density $\rho(z)=\Sigma \exp \left(-(z / H)^{2} / 2\right) /(\sqrt{2 \pi} H)$ ), the height $H_{\text {spall }}$ of this layer obeys

$\Sigma_{\text {spall }}=\frac{\Sigma}{2} \operatorname{erfc}\left(\frac{H_{\text {spall }}}{H \sqrt{2}}\right)$.

For $\Sigma \sim 10^{5 \pm 1} \mathrm{~kg} \mathrm{~m}^{-2}$ and $\Sigma_{\text {spall }}=10^{1 \pm 1} \mathrm{~kg} \mathrm{~m}^{-2}$, I have $\chi \equiv H_{\text {spall }} / H \approx 3 \pm 1$, so that

$\frac{H_{\text {spall }}}{R}=0.16\left(\frac{\chi}{3}\right)\left(\frac{R}{0.5 \mathrm{AU}}\right)^{1 / 2}\left(\frac{T}{1500 \mathrm{~K}}\right)^{1 / 2}$.

Since, for $x \gtrsim 2$, I have $^{10}$

$\operatorname{erfc}(x) \approx \frac{\exp \left(-x^{2}\right)}{\sqrt{\pi} x}$

the density at $z=H_{\text {spall }}$ may be approximated as

$\rho\left(H_{\text {spall }}\right)=\frac{\chi \Sigma_{\text {spall }}}{H}=8 \times 10^{-9} \mathrm{~kg} \mathrm{~m}^{-3}\left(\frac{\chi}{3}\right)\left(\frac{\Sigma_{\text {spall }}}{10 \mathrm{~kg} \mathrm{~m}^{-2}}\right)\left(\frac{1500 \mathrm{~K}}{T}\right)^{1 / 2}\left(\frac{0.5 \mathrm{AU}}{R}\right)^{3 / 2}$.

The nominal value (corresponding to a number density of $2 \times 10^{12} \mathrm{~cm}^{-3}$ ) is halfway in the range studied by Umebayashi \& Nakano (1981) so the neutron contribution (ignored in this paper) can be seen in their Figs. 2 and 5 to be limited. Then, the settling parameter (Jacquet et al. 2012) for a solid of density $\rho_{\mathrm{s}}$ and radius $a$ there is

$S_{z}\left(H_{\text {spall }}\right)=\sqrt{\frac{\pi}{8}} \frac{\rho_{\mathrm{s}} a}{\rho\left(H_{\text {spall }}\right) c_{\mathrm{s}} \delta_{z}} \approx \sqrt{\frac{\pi}{8}} \frac{\rho_{\mathrm{s}} a}{\Sigma_{\text {spall }} \delta_{z} \chi}=0.2\left(\frac{\rho_{\mathrm{s}} a}{1 \mathrm{~kg} \mathrm{~m}^{2}}\right)\left(\frac{10 \mathrm{~kg} \mathrm{~m}^{-2}}{\Sigma_{\text {spall }}}\right)\left(\frac{10^{-1}}{\delta_{z}}\right)\left(\frac{3}{\chi}\right)$,

with $\delta_{z}$ the vertical diffusion coefficient normalized to $c_{\mathrm{s}}^{2} / \Omega_{K}$ (which should be of order $\alpha$ ).

Simulations of ideal MHD turbulence indicate $\delta_{z} \sim \alpha \propto \rho^{-1}$ over a large extent of the disk thickness, although it should stall in the corona (e.g., Jacquet 2013, and references therein). Since $S_{z}$ may be expected to be $\lesssim 1$ there from the above evaluation, it may not be a dramatic underestimate to treat it as constant throughout the vertical thickness of the disk. Under that assumption, a population of identical solids has a density (Jacquet 2013)

$\rho_{\mathrm{c}}(z)=\frac{\Sigma_{\mathrm{c}} \sqrt{1+S_{z}}}{\sqrt{2 \pi} H} \exp \left(-\frac{S_{z}+1}{2}\left(\frac{z}{H}\right)^{2}\right)$,

with $\Sigma_{\mathrm{c}}$ the surface density of the population. The ${ }^{10} \mathrm{Be}$ production rate averaged over this distribution is then

$\left\langle\left(\frac{{ }^{10} \mathrm{Be}}{{ }^{9} \mathrm{Be}}\right)_{\mathrm{tg}}\right\rangle_{\rho_{\mathrm{c}}} \equiv \frac{2}{\Sigma_{\mathrm{c}}} \int_{0}^{+\infty} \mathrm{d} \Sigma_{\mathrm{c}}^{\prime}\left(\frac{{ }^{10} \mathrm{Be}}{{ }^{9} \mathrm{Be}}\right)_{\mathrm{tg},+}\left(\Sigma^{\prime}\left(\Sigma_{p}^{\prime}\right)\right)$,

with $\Sigma_{\mathrm{c}}^{\prime}$ and $\Sigma^{\prime}$ the columns of the solids and gas, respectively, integrated vertically from the surface. If I let

$\left(\frac{{ }^{10} \mathrm{Be}}{{ }^{9} \mathrm{Be}}\right)_{\mathrm{tg},+} \equiv\left(\frac{{ }^{10} \mathrm{Be}}{{ }^{9} \mathrm{Be}}\right)_{\mathrm{tg},+}(0) g\left(\frac{\Sigma^{\prime}}{\Sigma_{\text {spall }}}\right)$,

and since

$\Sigma^{\prime}=\frac{\Sigma}{2} \operatorname{erfc}\left(\frac{\operatorname{erfc}^{-1}\left(2 \Sigma_{\mathrm{c}}^{\prime} / \Sigma_{\mathrm{c}}\right)}{\sqrt{1+S_{z}}}\right)$,

this becomes

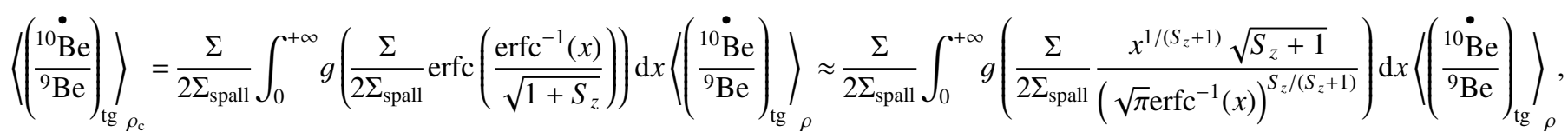

where I have used approximation (C.4), with the corollary $\operatorname{erfc}^{-1}(z) \approx \sqrt{-\ln \left(\sqrt{\pi} z \operatorname{erfc}^{-1}(z)\right)}$ for $z \ll 1$. Roughly speaking, the integrand only contributes $(\sim 1)$ for a $g$ argument $\lesssim 1$, that is $x \lesssim\left(\sqrt{\pi} \operatorname{erfc}^{-1}(x)\right)^{S_{z}}\left(2 \Sigma_{\text {spall }} / \Sigma \sqrt{S_{z}+1}\right)^{S_{z}+1}$ so that

$\left\langle\left(\frac{\dot{0}^{10} \mathrm{Be}}{\left({ }^{9} \mathrm{Be}\right.}\right)_{\operatorname{tg}}\right\rangle_{\rho_{\mathrm{c}}} \sim \frac{1}{\sqrt{S_{z}+1}}\left(\sqrt{\pi \ln \left(\Sigma / \Sigma_{\text {spall }}\right)} \frac{2 \Sigma_{\text {spall }}}{\Sigma}\right)^{S_{z}}\left\langle\left(\frac{{ }^{10} \mathrm{Be}}{\left({ }^{9} \mathrm{Be}\right.}\right)_{\mathrm{tg}}\right\rangle_{\rho}$.

So ${ }^{10}$ Be production should quickly drop as $S_{z}$ approaches unity and become negligible for $S_{z} \gtrsim 1$.

${ }_{10}$ This is because $\operatorname{erfc}^{\prime}(x)=-2 \exp \left(-x^{2}\right) /(\sqrt{\pi}) \sim-\exp \left(-x^{2}\right)\left(2+1 / x^{2}\right) / \sqrt{\pi}=\left(\exp \left(-x^{2}\right) /(\sqrt{\pi} x)\right)^{\prime}$. 